\title{
L'emploi public et les trajectoires d'insertion des jeunes
}

\author{
Vanessa di Paola et Stéphanie Moullet*
}

\begin{abstract}
Analyser le rôle de l'emploi dans le secteur public dans l'insertion des jeunes permet de mieux cerner qui sont les jeunes passant par ce secteur et d'évaluer l'importance de cet employeur dans leur parcours d'insertion. À partir d'un échantillon de jeunes sortis du système scolaire en 1998 et qui ont connu au moins un épisode d'emploi dans la fonction publique dans les trois années suivantes, neuf parcours types d'insertion ont été construits. Si ces trajectoires ne permettent pas de préjuger du rôle du passage par l'emploi dans le secteur public dans l'accès à un statut de fonctionnaire titulaire ou un CDI du secteur privé pour cette cohorte, elles permettent néanmoins d'éclairer la façon dont ces itinéraires se construisent différemment, en particulier pour les hommes et les femmes. On remarquera néanmoins que l'analyse porte sur une période de bonne conjoncture économique où l'emploi des jeunes a bénéficié aussi des créations massives d'emplois aidés dans le secteur public (les emplois jeunes sont créés en 1997).
\end{abstract}

Ainsi, les jeunes femmes de cette cohorte accèdent rapidement au marché interne de la fonction publique : elles sont en proportion plus élevée et ont une plus grande chance d'accéder au statut de fonctionnaire à l'issue de la formation initiale. À l'inverse, les jeunes hommes ont plus souvent des trajectoires où l'emploi public est tardif dans le parcours. Plus généralement, les trajectoires marquées par un passage assez long dans un emploi public sont davantage féminines, alors que les hommes ont une plus grande probabilité d'appartenir à des trajectoires dont l'état dominant est le CDD du secteur privé, l'emploi jeune ou le chômage. À cet effet vient s'ajouter celui du niveau de qualification, puisqu'il y a davantage de femmes diplômées dans les trajectoires d'insertion rapide dans le secteur public, et plus d'hommes diplômés dans celles où le secteur privé domine. Mais indépendamment de cette répartition, les jeunes des trajectoires passant par le secteur public ont des niveaux de qualification plus élevés. Cette typologie montre également la place occupée par l'emploi dans le secteur public contractuel dans l'insertion des jeunes, cet emploi ne débouchant pas toujours sur un CDI ou sur le statut de fonctionnaire.

Au-delà de la typologie obtenue, l'estimation des probabilités d'accès au statut de fonctionnaire d'une part et d'un CDI d'autre part, permet d'appréhender le rôle de l'emploi en CDD dans le secteur public. Ainsi, être passé par un emploi antérieur en CDD (de plus de 6 mois) accroît les chances de se stabiliser dans le secteur public si et seulement si cet emploi s'est effectué dans le secteur public. Une expérience d'emploi en CDD dans le secteur privé a, en revanche, moins de chances de conduire au statut de fonctionnaire.

* Vanessa di Paola appartient au Lest-Idep et Stéphanie Moullet au Céreq.

Les noms et dates entre parenthèses renvoient à la bibliographie en fin d'article. 
$\mathbf{S}$ avec un quart de la population active dans ses rangs, la fonction publique est, en France, le premier employeur, l'accent est rarement mis sur le rôle de l'emploi public dans l'insertion des jeunes. Pourtant, en 2001 , $14 \%$ de la population active de moins de 30 ans est employée dans la fonction publique d'État ou territoriale. L'importance de cet employeur, notamment du fait de sa taille, et les prochains départs massifs en retraite des agents de la fonction publique, et d'éventuelles difficultés de recrutement à venir, posent la question de son attractivité. Si cette attractivité intéresse l'employeur, du point de vue des jeunes, la question devient celle de l'usage qui peut être fait de l'emploi public dans leur parcours d'insertion professionnelle. La période étudiée, de 1998 à 2001 , correspond à une reprise de l'emploi associée à la création massive d'emplois aidés à destination des jeunes (création des emplois jeunes en 1997).

La spécificité d'une analyse économique de l'emploi public tient au mode de fonctionnement particulier du secteur public où coexistent un marché interne très spécifique sur lequel l'emploi est pratiquement garanti à vie et un marché externe dont on peut interroger le fonctionnement : est-il organisé comme un marché concurrentiel privé (CDD du secteur public) ou bien a-t-il pour objectif de se substituer à l'employeur privé pour les jeunes en difficulté (cas des mesures d'aide à l'insertion) ? Pour les entrants sur le marché du travail, les mobilités, selon leur fréquence et la nature des transitions en cause, peuvent traduire des parcours d'insertion où le passage par un emploi dans la fonction publique, quel que soit son statut, ne permet pas de stabilisation visible en emploi durable (en 2001, seule la moitié des jeunes en emploi dans la fonction publique se déclare fonctionnaire, alors que l'autre moitié se répartit sur une pluralité de statuts). À l'inverse, elles peuvent refléter une stratégie de construction de début de carrière professionnelle où l'emploi public tient une place déterminante. L'accès à un marché interne, celui de la fonction publique, pourrait dans ce cas relever de logiques non opportunistes. La complexification des parcours professionnels y prend une forme particulière : la carrière peut $\mathrm{y}$ débuter par des emplois précaires, coût d'entrée pour l'accès à un parcours « sécurisé ».

Dans cette perspective, à l'intersection des questions de l'insertion professionnelle et des recrutements des débutants par la fonction publique (le renouvellement de sa maind'œuvre), on s'attachera à donner des éléments de réponse aux questions suivantes : quelle est la nature de l'insertion professionnelle des jeunes qui connaissent un ou plusieurs emplois dans la fonction publique en début de vie active ? Du point de vue de la stratégie individuelle de construction de début de carrière professionnelle, quelle est l'efficacité du passage par un emploi dans la fonction publique? Quels sont les profils des jeunes pour lesquels ce passage conduit à une situation d'emploi stabilisée?

Pour ce faire, on analyse la place des séquences d'emploi public dans les trajectoires d'insertion. Ainsi, à partir des trois premières années de vie active, on construit des trajectoires types afin d'appréhender la spécificité de l'employeur public dans l'insertion des jeunes, quel que soit leur niveau de formation. On ne s'intéresse alors qu'aux individus ayant connu au moins un épisode d'emploi dans le secteur public. Les jeunes ont-ils un désir d'intégrer la fonction publique et mettent-ils en place des stratégies d'insertion sur le marché interne public? Si de telles stratégies existent, elles passent par le choix d'une formation permettant une entrée rapide en tant que fonctionnaire ou par un accès au marché interne par l'intermédiaire d'un emploi public non titulaire.

L'objectif de cet article est double : descriptif en proposant une typologie de parcours professionnels en début de vie active de la génération 1998 ; analytique en évaluant l'effet causal du passage par un emploi public, hors emplois aidés, sur l'obtention ultérieure d'un emploi à durée indéterminée dans les secteurs public (statut de fonctionnaire) ou privé. Dans quelle mesure l'accès au statut de fonctionnaire au terme des trois premières années de vie active est-il favorisé par le fait d'avoir connu préalablement un CDD du secteur public, plutôt que du secteur privé ?

Plus précisément, après avoir présenté les données utilisées, issues de l'enquête Génération 98 du Céreq, on établit la typologie des trajectoires professionnelles des jeunes expérimentant au moins un emploi dans la fonction publique, restreinte à la fonction publique 
d'État et territoriale (1). Afin de préciser le rôle des déterminants individuels dans les liens que les jeunes entretiennent avec la fonction publique, les déterminants de l'appartenance aux différents parcours types d'insertion sont ensuite identifiés. L'accent est enfin mis sur l'accès à un statut d'emploi pérenne : quels sont les facteurs explicatifs des probabilités d'emplois de fonctionnaire ou en CDI après trois ans de vie active et en particulier quel est le rôle des CDD des secteurs public et privé ?

\section{L'insertion professionnelle et l'enquête Génération 98}

L es données utilisées sont issues de l'enquête Génération 98 réalisée en mars 2001 par le Centre d'Études et de Recherches sur les Qualifications (Céreq). Elles concernent 55000 sortants du système éducatif en 1998 tous niveaux de formation et toutes spécialités de formation confondues parmi les 750000 primo-sortants à cette date. Les données sont rétrospectives et portent sur la période de janvier 1998 à mars 2001. Elles permettent d'analyser les trois premières années de vie active au regard de la formation initial (2). Elles autorisent donc la comparaison des modalités d'insertion professionnelle des jeunes selon leur niveau d'éducation dans une même conjoncture économique (cf. encadré 1).

\section{Les jeunes et le secteur public}

Afin d'identifier la singularité des jeunes expérimentant un emploi dans le secteur public au cours de leurs premières années de vie active, on construit un sous-échantillon composé de jeunes ayant connu l'emploi public (échantillon dit « secteur public »), ce qui permet de mettre en évidence les particularités de cette population comparativement à l'échantillon global (la population de Génération 98).

Le sous-échantillon « secteur public » concerne uniquement les individus (et les informations relatives aux séquences d'emploi et de nonemploi) dont au moins un emploi, d'une durée minimale d'un mois sur la période d'observation, s'exerce dans la fonction publique d'État ou territoriale, quel que soit son statut.

Alors que la population des jeunes sortant du système éducatif en 1998 est pour 51 \% compo- sée d'hommes, l'échantillon des jeunes ayant exercé au moins un emploi dans le secteur public est majoritairement féminin $(59 \%$ de femmes, répartition identique dans les deux fonctions publiques) (cf. annexe 1). La prédominance des femmes dans le sectur public se constate donc chez les jeunes comme pour l'ensemble de la population active (Audier, 2000).

La répartition des niveaux de formation (3) au sein de l'échantillon diffère de celle observée dans la population totale des sortants du système éducatif (cf. annexe 1) : le niveau II, avec $30 \%$ de l'effectif, est le plus représenté (contre le niveau Vbis pour l'ensemble de la population) ; ce niveau étant celui des enseignants et donc en lien avec la part des emplois occupés dans l'Éducation nationale (34\% des emplois du secteur public en mars 2001 dans l'enquête). Les sortants de formation initiale détenteurs d'un diplôme supérieur à bac +2 sont surreprésentés au sein de l'échantillon « secteur public » relativement à l'ensemble des sortants $(38 \%$ contre environ $19 \%$ ).

Le poids des formés de niveau IV + $(17 \%)$, plus nombreux que les niveaux IV (12\%), est une caractéristique notable de l'échantillon « secteur public » dans la mesure où les ordres de grandeur sont inversés dans la population globale $(12,3 \%$ de niveau IV + , contre $17,4 \%$ de niveau IV). Ce poids des jeunes sortants de l'enseignement supérieur sans en être diplômé pourrait être dû aux jeunes qui décident finalement de passer un concours de la fonction publique. Cette hypothèse pourra être vérifiée dans l'analyse des trajectoires d'insertion.

La structure des niveaux de formation diffère entre fonction publique d'État et territoriale. Si $48 \%$ des jeunes en emploi dans la fonction

1. L'exclusion de la fonction publique hospitalière dans une étude relative au rôle de l'emploi public dans l'insertion des jeunes peut surprendre. II s'agit en fait d'un choix pragmatique dû à la difficulté de distinguer hôpitaux publics et privés dans l'enquête. Mais ce choix peut également s'expliquer lorsqu'on s'intéresse aux entrants sur le marché du travail puisqu'en effet la fonction publique hospitalière a des canaux de recrutement spécifiques. II y a non-substitution entre cette fonction publique et les autres fonctions publiques étant donnée la singularité des métiers qu'elle concerne (il n'y aurait que les entrées comme directeurs d'hôpitaux qui draineraient le même public que les autres fonctions publiques). Cet argument pourrait s'appliquer aux enseignants et donc justifier leur exclusion de l'analyse. On a choisi de les conserver afin de ne pas morceler la fonction publique d'État.

2. Cette enquête succède à l'enquête Génération 92 qui interroge cinq ans après la fin de leur formation initiale les jeunes sortis du système éducatif en 1992. Si l'objectif poursuivi est le même, Génération 92 met davantage l'accent sur les situations d'emploi que ne le fait Génération 98 (Bref, 181, p. 7). Concernant cette dernière, des ré-interrogations sont prévues en 2003 et 2005.

3. Voir en annexe 1 la nomenclature des niveaux de formation initiale. 
publique d'État en mars 2001 sont de niveaux I et II de formation, ils sont seulement $22 \%$ dans ce cas pour la fonction publique territoriale.

Les caractéristiques familiales mettent surtout en avant une meilleure connaissance de la fonc- tion publique pour les jeunes de l'échantillon «secteur public» que pour l'ensemble de l'effectif. En effet, ils sont plus nombreux à avoir un conjoint $(31 \%$ contre $21,5 \%)$, un père $(27 \%$ contre $23 \%)$ ou une mère $(28 \%$ contre $23 \%$ ) qui travaille dans le secteur public. Cette proximité familiale est nécessairement anté-

Encadré 1

\section{L'EMPLOI DANS LE SECTEUR PUBLIC ET L'ENQUÊTE GÉNÉRATION 98}

L'enquête a pour objet de rendre compte des différentes composantes des parcours d'insertion professionnelle : elle fournit des informations individuelles, sociodémographiques et relatives au parcours scolaire ainsi qu'aux différentes séquences d'emploi ou de nonemploi. Une séquence étant définie comme une durée passée dans un état, une séquence d'emploi correspond à une période d'emploi exercée au sein d'un même établissement sans interruption. Les séquences de non-emploi correspondent à des durées de formation (ou de reprise d'études), d'inactivité (y compris le Service national) et de recherche d'emploi. L'ensemble de ces situations datées est synthétisé au travers du calendrier professionnel renseignant sur tous les états des individus interrogés, mois par mois, de janvier 1998 à mars 2001.

Pour chaque séquence d'emploi, le secteur d'activité, la PCS occupée, le contrat et le temps de travail ainsi que le salaire mensuel perçu, primes incluses, sont connus. La variable décrivant le secteur - public ou privé - de l'emploi occupé est également disponible et relève d'une déclaration individuelle (1). À partir de cette dernière et sur la base du nom déclaré de l'employeur, il devient possible de distinguer les trois fonctions publiques - fonction publique d'État, territoriale et hospitalière -, et au sein de la fonction publique d'État, de séparer les types d'employeur (Éducation nationale, Armées et autres ministères). Les entreprises publiques nationalisées sont également identifiables.

La définition de l'emploi public est ici fondée sur la nature juridique de l'employeur (2) et non pas sur le statut juridique - droit public, droit privé - des salariés; le terme de secteur public ne se limite pas au statut de fonctionnaire mais à l'ensemble des contrats de travail proposé par l'employeur public (3). In fine, une variable est construite précisant pour chaque épisode d'emploi connu par l'individu, et quel que soit le statut de cet emploi (CDD, CDI, intérim, etc.), s'il s'agit d'un emploi « public » (fonction publique d'État ou territoriale) ou " privé ".

\section{Les limites de l'enquête}

La rareté de travaux portant sur l'employeur public est certainement en partie due à la difficulté de spécifier le champ « secteur public ». Pour ce qui nous concerne, si les données sur le cheminement au cours des premières années de vie active permettent l'analyse des parcours d'insertion professionnelle, elles présentent elles aussi, dans une certaine mesure, des limites par rapport à l'analyse de l'emploi public.

La première limite imposée par les données tient au défaut de couverture de la base de sondage des sortants du système éducatif : un ensemble d'écoles de la fonction publique, essentiellement celles qui relèvent du ministère de l'Économie et des finances, de la Défense, de la Justice, du Premier ministre, en sont exclues. Ces écoles préparent massivement à des emplois de catégorie $A$, bien qu'au sein de ces dernières, coexistent des élèves fonctionnaires (qui seront nécessairement fonctionnaires à la sortie) et des étudiants qui se destinent à une carrière dans le secteur privé. La base de données ne contient donc aucun agent de catégorie $\mathrm{A}$ à la sortie de ces écoles. Pour les écoles incluses dans la base de sondage, on a toutes les raisons de penser qu'elles ne sont pas représentatives des écoles non enquêtées, lorsqu'elles appartiennent à des ministères différents (l'hétérogénéité des écoles appartenant à un même ministère rend la pondération éventuelle peu fiable). L'analyse de l'insertion dans la fonction publique ne peut porter sur les caractéristiques de ceux qui entrent dans la fonction publique en tant que fonctionnaire à la suite d'une scolarité suivie dans une école de la fonction publique.

La deuxième limite concerne certains individus élèves fonctionnaires (30 individus) au cours de la période d'observation (1998-2001). On choisit de les considérer en formation initiale et non en emploi dans la fonction publique : le temps d'école est un temps de formation, ce qui permet d'éviter de sous-évaluer le niveau de formation initiale des fonctionnaires.

\footnotetext{
1. Le secteur d'activité est issu de la réponse à la question "L'entreprise dans laquelle vous travaillez appartient-elle: a) au secteur privé, b) au secteur public, c) ne sait pas. " ainsi que des recodages sur la base notamment du nom de l'employeur. 2. Cette nature juridique de l'employeur étant mal connue, la définition de l'emploi public a fait l'objet de nombreux recodages sur la base des informations recueillies sur l'employeur. 3. La notion d'emploi public n'a pas de définition stabilisée, son périmètre et son contenu étant souvent imprécis (ou non précisés). Le rapport annuel de l'Observatoire de l'emploi public (2001) permet de clarifier ses différents contours possibles. Au-delà du noyau central constitué de l'ensemble des trois fonctions publiques, le dénombrement se heurte à des problèmes de définition du champ et à des contraintes imposées par les sources statistiques (Brenot-Ouldali, 1999). Dans la mesure où les études relatives au secteur public utilisent des sources différentes et donc recouvrent des champs différents, les comparaisons des résultats s'en trouvent limitées.
} 
rieure à l'occupation de l'emploi pour ce qui concerne les parents (puisqu'il s'agit de l'emploi parental occupé à la fin des études du jeune), sans que ce soit toujours le cas pour la position du conjoint.

Les variables de parcours traduisent une plus grande mobilité d'employeur dans la population connaissant au moins un épisode dans le secteur public, que dans la population totale. On pourra par la suite chercher à vérifier qu'il s'agit là d'un effet «emploi d'attente » avant de réussir à un concours de la fonction publique ou encore du passage par les mesures publiques d'aide à l'insertion (donc passage par un emploi public) qui ont tendance à concerner des jeunes au parcours « instable».

Trois ans après la sortie du système éducatif, près de $52 \%$ de l'effectif global occupe un emploi pérenne soit en tant que fonctionnaire $(7,8 \%)$ soit en CDI du secteur privé $(44,5 \%)$, alors que dans l'échantillon «secteur public » les jeunes sont dans $35 \%$ des cas fonctionnaires et en CDI du secteur privé dans $10 \%$ des situations.

Enfin, la population des jeunes passés au moins une fois par le secteur public est, trois ans après la sortie du système éducatif, composée majoritairement de personnels de l'Éducation nationale (34\%). Le poids de l'emploi dans les collectivités territoriales est également à souligner puisqu'il concerne $21 \%$ des jeunes de l'échantillon « secteur public ».

\section{Les passages par l'emploi dans le secteur public}

Afin de mettre en évidence l'effet propre des caractéristiques toutes choses égales par ailleurs, on estime la probabilité d'appartenir à l'échantillon «secteur public» (cf. annexe 2). Les résultats d'estimation sont conformes aux statistiques descriptives. En effet, être un homme agit négativement sur la probabilité d'occuper un emploi public de janvier 1998 à mars 2001, tous statuts confondus. Toutes choses égales par ailleurs, les jeunes titulaires d'un diplôme de formation de niveau II ou de niveau IV + connaissent des probabilités de sélection plus fortes que les autres diplômés. La proximité familiale avec la fonction publique (l'appartenance de l'emploi des parents ou du conjoint à ce secteur) joue dans le même sens. Le pays d'origine, s'il est autre que la France, diminue la probabilité de connaître au moins une séquence d'emploi public (4). Enfin, résider ailleurs qu'à Paris à la fin des études accroît la probabilité de sélection: cet effet renvoie à l'état relatif du marché du travail entre Paris et la province, l'emploi dans le secteur privé étant plus dynamique dans la région parisienne, le secteur public est donc relativement plus important dans les autres régions.

En résumé, la probabilité d'appartenir à l'échantillon « secteur public » est d'autant plus grande que l'individu est une femme, de niveau II ou IV + de formation initiale, ayant une proximité familiale avec le secteur public, d'origine française et résidant en province.

\section{Emploi public et typologies des parcours professionnels}

L es caractéristiques des jeunes ayant connu l'emploi dans le secteur public étant mieux cernées, on peut s'intéresser plus précisément aux trajectoires d'insertion des jeunes sortis du système scolaire en 1998. Les trajectoires sont construites à partir de l'échantillon «secteur public », c'est-à-dire celui composé de jeunes passés au moins une fois par un emploi de la fonction publique sur les trois années d'observation.

\section{Construction de la typologie de trajectoires}

À partir des calendriers professionnels des jeunes entrant sur le marché du travail en 1998, on repère les événements caractéristiques de leurs parcours d'insertion. L'existence de trajectoires constituées de passages différents en termes de contrat de travail et de durée conduisent à penser que tous les jeunes ne font pas les mêmes usages de l'emploi dans le secteur public.

Une analyse des chronogrammes ou trajectoires selon la méthode d'Espinasse (1994) permet de distinguer les événements caractéristiques du parcours (CDD, CDI, chômage, etc.) ainsi que, et c'est là l'intérêt de cette méthode, la durée de ces événements. Elle ne pose pas d'hypothèse $a$ priori sur la nature et la hiérarchie des différents états entre eux (elle ne retient aucune autre information que celles disponibles dans les chronogrammes), mais considère la séquentia-

4. Le statut de fonctionnaire nécessitant la nationalité française (à quelques exceptions près). 
lité des états. On obtient ainsi une description des parcours sur le marché du travail qui n'a trait qu'aux événements survenus sur ce marché, aucun facteur explicatif de ces trajectoires n'étant envisagé à ce stade. Ces derniers seront l'objet d'une analyse spécifique (cf. infra). Cette démarche en deux étapes (construction d'une typologie puis identification des facteurs explicatifs de la probabilité d'appartenance aux différents parcours types) peut être rapprochée d'une procédure tendant à retranscrire une dépendance temporelle dans un premier temps, indépendamment de toute hétérogénéité individuelle ; puis à s'intéresser au poids de l'hétérogénéité individuelle dans la construction des parcours d'insertion. On sait que la distinction entre dépendance temporelle et hétérogénéité individuelle est essentielle mais souvent difficile à identifier dans la modélisation économétrique, en particulier dans les modèles de durée (di Paola, 2000).

L'objectif de la méthode de classification des trajectoires retenues est de synthétiser la diversité des trajectoires individuelles d'insertion à l'aide de classes de trajectoires homogènes (cf. encadré 2). Il s'agit d'exploiter la dimension longitudinale des informations : 40 mois d'observation décrivent la succession des épisodes relatifs à la position occupée sur le marché du travail, et notamment la succession éventuelle d'épisodes d'emploi, définis par un changement d'établissement et non pas de profession ou de contrat de travail.

Les différents états, retenus sur la base conjointe du secteur d'emploi et du statut du contrat de travail pour les situations d'emploi, sont les suivants (5) : 1) Fonctionnaire, 2) CDI du secteur privé, 3) CDD, contrat saisonnier ou contrat d'intérim du secteur public, 4) CDD, contrat saisonnier ou contrat d'intérim du secteur privé, 5) Emplois aidés du secteur public (emplois jeunes, contrat de qualification, contrat d'apprentissage, CES), 6) Emplois aidés du secteur privé (emplois jeunes, contrat de qualification, contrat d'apprentissage), 7) Chômage, 8) Formation initiale, inactivité, reprise d'études ou Service national.

La procédure de construction de la typologie aboutit à neuf trajectoires types que l'on peut caractériser par des états dominants et/ou une (in)stabilité professionnelle sur les trois années

5. Précisons que, alors que les statistiques descriptives laissaient penser que la distinction entre les deux fonctions publiques - d'Etat et territoriale - dans la constitution des trajectoires types était pertinente, l'exercice n'a pas pour autant conduit à des résultats autres que ceux développés dans la suite de cette analyse.

Encadré 2

\section{LA MÉTHODE DES TRAJECTOIRES TYPES}

La chronologie des états, leur durée et leur enchaînement constituent la trajectoire professionnelle d'un jeune entrant sur le marché du travail. Elle est a priori aussi unique que lui. Mais certaines trajectoires sont plus proches que d'autres. La recherche de trajectoires types suppose que l'on regroupe entre elles les trajectoires homogènes. Cela nécessite de mesurer la distance entre elles. C'est l'information séquentielle des chronogrammes individuels qui permet de mesurer cette distance entre les trajectoires individuelles : elle est égale au nombre de mois où les individus associés connaissent des situations différentes, elle est nulle si les états sont identiques pour le même mois. Cette méthode repose ainsi sur une distance calculée non pas entre des groupes d'individus mais entre les trajectoires elles-mêmes (Beduwé, Dauty et Espinasse, 1995).

L'étape suivante consiste à identifier les pôles où se concentrent les trajectoires similaires ou voisines : une classification automatique des individus sur la base des distances calculées établit des classes cohérentes et indique pour chacune les états dominants et leurs durées. Les classes sont telles que leur variance intra est minimale et la variance entre elles maximale. Les effectifs de différentes classes ainsi que la durée moyenne passée dans les états permettent de définir alors le nombre pertinent de classes. Cette étape aboutit donc à des trajectoires types et indique pour chacune les états dominants et les durées moyennes. L'ordre d'enchaînement des situations se visualise au travers d'une représentation graphique par trajectoire type (Béduwé et Cahuzac, 1997).

D'autres méthodes de classification des trajectoires poursuivant le même objectif existent et ont été comparées par Espinasse et Giret (1996). La méthode retenue a pour principal intérêt de n'imposer aucune hypothèse sur la nature, le recodage ou la hiérarchie des états entre eux. Elle utilise toute l'information disponible sur les cheminements individuels dans leur forme d'origine, à l'exclusion de toute autre variable. En revanche, elle fait implicitement l'hypothèse que tous les coûts de passage d'un état à un autre sont égaux à 1 (elle ignore les différentes probabilités de transition d'un état à un autre). 
d'observation, c'est-à-dire l'existence de discontinuité de l'emploi ou de mobilité professionnelle. Ces parcours types ne préjugent pas de l'état final (celui de la date d'enquête) occupé par un individu donné mais renseignent sur la situation finale la plus fréquemment observée pour l'ensemble des individus d'une même trajectoire.

Il est usuel, à partir de cette méthode, de caractériser chacune des trajectoires types par les états dominants (cf. tableau 1 et graphiques en annexe 3).

La $1^{\text {re }}$ trajectoire concerne les jeunes accédant rapidement après leur sortie de formation initiale à un emploi de fonctionnaire.

La $2^{\mathrm{e}}$ trajectoire regroupe les individus qui, après quelques mois sur le marché du travail passé au chômage, puis en CDD du public ou du privé, occupent majoritairement un CDI du secteur privé (66\% sont en CDI en mars 2001).

La $3^{\mathrm{e}}$ trajectoire a trait à des individus au parcours relativement instable: chômage puis CDD (ou intérim) (6) du public puis CDD du privé, ce dernier étant l'état caractéristique de la trajectoire.

La $4^{\mathrm{e}}$ trajectoire renvoie à un parcours relativement instable passant par du chômage, puis par un CDD du secteur privé, pour enfin transiter vers un CDD du secteur public.

La $5^{\mathrm{e}}$ trajectoire est relative au passage par du chômage de longue durée débutant dès la sortie du système éducatif, et jusqu'en juin 2000 , la sortie du chômage se faisant principalement vers le secteur public, les emplois stables ou instables.
La $6^{\mathrm{e}}$ trajectoire regroupe essentiellement des jeunes qui vont soit continuer à se former, soit être inactifs jusqu'en juin 2000, puis vont accéder pour $42 \%$ d'entre eux à un statut de fonctionnaire, les autres se répartissant essentiellement entre les emplois instables (emplois aidés privés ou publics, CDD).

La $7 \mathrm{e}$ trajectoire concerne des individus connaissant un passage par un CDD du secteur privé à leur sortie de formation initiale, CDD du privé d'insertion puisqu'ils transitent ensuite fréquemment vers un emploi de fonctionnaire ou, dans une moindre mesure, vers un CDI du secteur privé.

La $8^{\mathrm{e}}$ trajectoire trace un parcours assez long en CDD du secteur public, auquel les jeunes accèdent rapidement après la sortie de formation initiale, et que $40 \%$ quittent autour du $31^{\mathrm{e}}$ mois, majoritairement pour un emploi de fonctionnaire $(15 \%)$.

La $9^{\mathrm{e}}$ trajectoire est constituée des emplois jeunes, auxquels les individus accèdent après une période de chômage et d'emploi instable (CDD du privé).

\section{Trajectoires et secteur public}

La matrice des durées moyennes permet d'identifier les caractéristiques de chacune des trajectoires et de différencier de manière plus nette la

6. L'analyse des durées moyennes cumulées dans différents états désagrégés par trajectoire type permettra de préciser l'importance des CDD par rapport à l'intérim (ou inversement). II en sera de même pour chaque statut agrégé dans la typologie (mesures d'aide à l'insertion, etc.). Ainsi, il faut comprendre ici lorsqu'il est question de $C D D$, qu'il peut s'agir également d'un $C D D$ intérimaire.

Tableau 1

Trajectoires types

\begin{tabular}{|c|l|r|r|}
\hline Trajectoires types & \multicolumn{1}{|c|}{ Dénominations } & Fréquence (en \%) & Effectifs pondérés \\
\hline 1 & Fonctionnaires & 24,4 & 27367 \\
3 & CDI privé & 7,5 & 8425 \\
4 & CDD privé, instable & 6,4 & 7133 \\
5 & CDD public, instable & 7,4 & 8337 \\
6 & Chômage & 9,5 & 10686 \\
7 & Inactifs & 11,0 & 11420 \\
8 & CDD privé insérant & 6,3 & 7112 \\
9 & CDD public & 12,4 & 13883 \\
\hline
\end{tabular}

Champ : échantillon " secteur public ».

Source : Génération 98. 
classification obtenue. En particulier, cette matrice permet d'évaluer, dans une certaine mesure, la place de l'emploi public dans l'insertion des jeunes : il convient en effet de s'intéresser aux durées cumulées moyennes dans les différents statuts d'emploi public pour les trajectoires favorisant l'insertion, comparativement à ces mêmes durées dans les trajectoires « instables », dans les trajectoires à dominante «publique », comparativement à celles du «privé ». Il est aussi possible de qualifier l'insertion des jeunes ayant connu l'emploi public (secteur d'activité en mars 2001, type de contrat de travail, etc.).

Avant de s'intéresser aux trajectoires, notons que, sur l'ensemble de l'échantillon «secteur public », c'est la durée cumulée en tant que fonctionnaire qui est la plus longue (environ 8 mois). Sur les 40 mois d'observation, les individus passent près de $30 \%$ du temps dans le secteur public comme non titulaires (cette appellation regroupe l'ensemble des statuts du secteur public hormis celui de fonctionnaire), $20 \%$ en emploi titulaire et $15 \%$ en emploi privé.

Trajectoires stables et emploi dans le secteur public

Les trajectoires dites stables regroupent les trajectoires dont l'état dominant est un emploi sta- ble : trajectoires «fonctionnaires » et «CDI privé ».

L'analyse des durées permet de différencier et de caractériser chacune des trajectoires (cf. tableau 2). Ainsi, la trajectoire «fonctionnaire» est marquée par une longue durée en emploi de fonctionnaire, mais en dehors de ce statut, l'emploi public non titulaire est plus important que la part de l'emploi privé (y compris CDI). Cette trajectoire très stable est donc caractérisée par un accès rapide à la fonction publique sans avoir passé de temps conséquent dans des emplois privés.

Le symétrique de cette trajectoire pour le secteur privé est l'insertion via l'obtention d'un CDI du secteur privé. La trajectoire «CDI privé » est marquée, quant à elle, par une durée assez significative en emploi public (l'échantillon sélectionné concerne en effet les individus qui ont tous expérimenté au moins un épisode d'emploi public). Ainsi, passer par un CDD du secteur public peut être une étape (3,5 mois en moyenne) avant d'accéder à un CDI du secteur privé, le poids de cette durée dans l'emploi public temporaire est même plus important que celui des CDD du secteur privé.

Outre ces deux trajectoires particulièrement stabilisées, on peut considérer les trajectoires

Tableau 2

Matrice des durées moyennes

\begin{tabular}{|c|c|c|c|c|c|c|c|c|c|c|}
\hline \multirow{2}{*}{$\begin{array}{r}\text { États sur le marché du travail } \\
\text { Durées cumulées } \\
\text { moyennes (en mois) }\end{array}$} & \multicolumn{10}{|c|}{ Trajectoires types (1) } \\
\hline & 1 & 2 & 3 & 4 & 5 & 6 & 7 & 8 & 9 & $\begin{array}{l}\text { Échantillon } \\
\text { "secteur } \\
\text { public" }\end{array}$ \\
\hline Fonctionnaire & 27,12 & 1,49 & 0,75 & 0,55 & 1,43 & 4,20 & 2,22 & 1,21 & 0,32 & 7,72 \\
\hline CDI du privé & 0,41 & 18,61 & 1,86 & 1,18 & 0,81 & 0,98 & 1,06 & 0,51 & 0,57 & 2,10 \\
\hline CDD du public & 1,23 & 3,46 & 3,86 & 16,75 & 2,50 & 2,58 & 2,85 & 26,43 & 0,45 & 6,07 \\
\hline CDD du privé & 0,50 & 2,44 & 10,45 & 1,89 & 1,60 & 1,37 & 11,52 & 0,65 & 0,85 & 2,35 \\
\hline Intérim du public & 0,01 & 0,42 & 0,25 & 0,46 & 0,17 & 0,03 & 0,11 & 0,43 & 0,01 & 0,16 \\
\hline Intérim du privé & 0,26 & 0,63 & 4,18 & 1,01 & 0,53 & 0,96 & 5,42 & 0,33 & 0,55 & 1,07 \\
\hline Emploi jeune du public & 0,22 & 0,65 & 0,73 & 0,28 & 1,82 & 1,09 & 2,00 & 0,06 & 24,08 & 4,40 \\
\hline Emploi jeune du privé & 0,08 & 0,19 & 0,14 & 0,18 & 1,44 & 0,10 & 0,48 & 0,25 & 0,08 & 0,27 \\
\hline CES & 0,08 & 0,10 & 0,37 & 0,06 & 1,57 & 0,30 & 0,13 & 0,02 & 1,11 & 0,40 \\
\hline Apprentissage, CA, CQ public & 0,13 & 0,09 & 0,14 & 0,04 & 0,21 & 0,36 & 0,29 & 0,05 & 0,49 & 0,21 \\
\hline Apprentissage, CA, CQ privé & 0,05 & 0,25 & 0,63 & 0,33 & 0,80 & 0,21 & 0,72 & 0,14 & 0,07 & 0,27 \\
\hline Chômage & 0,73 & 2,64 & 4,86 & 4,30 & 18,25 & 2,04 & 2,66 & 1,47 & 2,89 & 3,76 \\
\hline Inactivité & 1,59 & 1,23 & 1,68 & 2,24 & 0,96 & 6,23 & 1,32 & 0,97 & 0,95 & 1,83 \\
\hline Formation & 6,45 & 6,63 & 7,39 & 7,34 & 7,11 & 12,66 & 7,01 & 6,48 & 6,46 & 7,32 \\
\hline
\end{tabular}

Lecture : les individus passés au moins une fois par un emploi public et appartenant à la trajectoire "fonctionnaires " ont passé en moyenne 27,12 mois en tant que fonctionnaires et 1,23 mois en CDD du secteur public, ces durées n'étant pas nécessairement continues. Champ : échantillon "secteur public ».

Source : Génération 98. 
pour lesquelles l'emploi public occupé tout au long du parcours a plutôt un rôle d'insertion (c'est le secteur d'emploi occupé en fin de période d'observation) et au contraire celles dans lesquelles l'emploi public expérimenté n'est pas associé à une situation stabilisée au terme des trois années suivant la sortie du système éducatif.

\section{Trajectoires d'insertion dans le secteur public}

Les trajectoires d'insertion dans le secteur public concernent les parcours chaotiques dont l'issue après trois années d'observation se fait majoritairement dans le secteur public : trajectoires « chômage », « inactifs », « CDD privé insérant ».

La trajectoire «CDD privé insérant » est assez atypique puisqu'il s'agit d'un parcours où l'état principalement occupé est celui de CDD dans le secteur privé en début de parcours, mais au terme de trois ans, l'emploi occupé, quel que soit son statut, se trouve le plus fréquemment dans le secteur public. C'est donc le passage par un CDD du secteur privé qui conduit à une insertion dans le secteur public $(25 \%$ des jeunes de cette trajectoire sont fonctionnaires en mars 2001). Ainsi, l'emploi public expérimenté ici n'est pas un passage permettant une insertion dans l'un ou l'autre des secteurs du marché du travail, mais une issue au CDD du secteur privé.

Deux autres trajectoires présentent une caractéristique similaire quant au rôle apparent de l'emploi public : les trajectoires « inactifs » et «chômage ». Dans les deux cas, les durées cumulées en emplois dans le secteur public ont un poids bien plus grand que celles en emplois dans le secteur privé. En particulier, dans la trajectoire « inactifs », où l'inactivité (y compris la formation) occupe une place bien sûr prépondérante, on constate un rôle d'insertion de l'emploi public particulièrement fort puisqu'à l'issue du parcours observé, $42 \%$ des jeunes sont fonctionnaires. La trajectoire « chômage », quant à elle, est caractérisée par un chômage de longue durée (18 mois en moyenne). Pour autant, la sortie du chômage sur les trois années d'observation est sensible et se fait en grande partie vers de l'emploi public. Ainsi, la durée passée en emploi public occupe $16 \%$ du temps d'observation pour l'emploi public instable et $3,5 \%$ pour l'état de fonctionnaire contre seulement $12 \%$ dans l'emploi privé ( $10 \%$ en CDD, $2 \%$ en CDI).

\section{Trajectoires instables et emploi public}

Les trajectoires qualifiées d'instables regroupent les trajectoires les plus chaotiques : trajectoires «CDD privé instable », « CDD public instable ».

Pour certaines trajectoires, l'emploi dans le secteur public ne semble pas avoir joué de rôle d'insertion. En effet, dans la trajectoire «CDD public instable », les CDD de ce secteur ne sont pas absorbés par le statut de fonctionnaire en mars 2001. Ainsi, le statut de fonctionnaire n'est pas dominant par rapport au CDI ni même au CDD du secteur privé. Les jeunes expérimentent une longue durée d'emploi en CDD du secteur public (26 mois en moyenne) sans pour autant se stabiliser au terme des trois années d'observation.

Le processus est quelque peu différent dans la trajectoire «CDD privé instable » où l'expérimentation importante de l'emploi public est concentrée en début de trajectoire. Ici, l'emploi dans le secteur public a un poids important mais ne conduit pas à une stabilisation au regard de l'état finalement occupé (les emplois de fonctionnaire ou en CDI ne sont pas majoritaires).

\section{Trajectoires où la stabilisation est inconnue et emploi dans le secteur public}

Les trajectoires à stabilisation inconnue rassemblent les parcours caractérisés par des états dont l'issue n'est pas observée : trajectoires « emploi jeunes », « CDD public ».

Pour les trajectoires «emploi jeune » et «CDD public instable », il est difficile d'analyser le rôle de l'emploi public dans l'insertion puisque au terme des premières années de vie active, c'est l'emploi jeune ou le CDD du secteur public qui domine massivement sans que l'on ait d'information sur une stabilisation ou non à son issue, celle-ci n'étant pas observée (7). En effet, les emplois jeunes sont pour la trajectoire éponyme l'état d'insertion à la fin des trois années d'observation pour $84 \%$ des jeunes de la trajectoire, et ces emplois jeunes sont expérimentés après quelques temps passés surtout au chômage (moins de 3 mois). De même, dans la trajectoire «CDD public », le CDD public est l'emploi final pour $79 \%$ des jeunes, avec une durée cumulée moyenne de 26 mois. Ceci est lié à la durée fréquente de trois années pour les CDD du secteur public, l'issue en est donc inob-

7. La durée des emplois jeunes est de cinq années. 
servée, comme celle des emplois jeunes. Les états précédant cette « insertion instable » sont le chômage puis le CDD du secteur privé. La question du rôle de ce type de contrat temporaire dans la trajectoire professionnelle à venir des jeunes reste posée.

Il s'agit ici d'une analyse factuelle informant sur le temps passé dans l'emploi public selon les trajectoires types. Pour autant, il convient de ne pas tirer de conclusion en termes de causalité : le fait que les jeunes de la trajectoire «CDI privé » connaissent une durée plus longue en CDD du secteur public qu'en CDD du secteur privé ne peut être pris comme preuve que le CDD public mène, plus favorablement qu'un CDD privé, à un CDI privé. En effet, dans cette trajectoire, une part non négligeable d'individus est fonctionnaire à la fin de l'observation (12\%) et on ne peut statuer sur la base des trajectoires types s'il s'agit des individus préalablement passés par un CDD public davantage que ceux finalement en CDI du privé $(66 \%)$. Seule une analyse économétrique modélisant les transitions pourrait permettre d'aller plus avant.

\section{Trajectoires et caractéristiques individuelles}

\section{Trajectoire et genre}

La composition sexuée de la population se différencie selon les trajectoires (cf. annexe 4, tableau A). Les parcours caractérisés par un passage assez long dans l'emploi public (trajectoire «fonctionnaire », «emploi jeunes », «CDD public ») sont davantage féminines (resp. $61 \%$, $68 \%, 62 \%$ ). Au-delà de ce premier constat, les statistiques descriptives par trajectoire mettent en évidence le fait que si les femmes diplômées ont une forte propension à entrer dans la fonction publique, les hommes de même niveau de diplôme s'orientent davantage vers le secteur privé sans expérimenter d'emplois dans le secteur public. Ainsi, à l'inverse de ce qui est généralement observé, les hommes se retrouvent ici surreprésentés dans les trajectoires plus instables («chômage» - $66 \%$ d'hommes, « inactifs» $-57 \%$ d'hommes, «CDD public instables »- $54 \%$ d'hommes). Pour leur part, les femmes sont majoritaires dans les trajectoires caractérisées par l'emploi public, à l'exception de la trajectoire « CDD public instables ».

Ainsi, l'analyse des trajectoires des individus passés au moins une fois par le secteur public permet de faire apparaître un phénomène d'anti- sélection: les hommes de l'échantillon « secteur public » passent plus souvent dans des emplois temporaires et s'insèrent moins dans des emplois stables que dans l'échantillon global. En revanche, les femmes s'insèrent plutôt mieux que dans l'échantillon global : le secteur public attire les femmes sur des statuts stables ou des trajectoires insérantes. L'ensemble de ces premiers résultats est à mettre en relation avec le niveau de diplôme et sera à analyser à partir des estimations économétriques.

\section{Trajectoire et famille}

On cherche à savoir ici si les trajectoires que l'on peut qualifier de « publiques », se singularisent par un entourage familial appartenant au secteur public. En particulier, le rôle joué par le statut des parents dans sa transmission aux enfants a précédemment été mis en évidence par Audier (2000) et mérite d'être étudié. Sur la base des simples statistiques descriptives, le secteur d'activité du père des jeunes dont la trajectoire est à dominante «publique » n'est que très peu différent de celui de la population totale et ne semble pas être une caractéristique discriminante des populations de chaque trajectoire. Le statut d'emploi de la mère est davantage différencié. En effet, si transmission du statut il y $a$, il passerait davantage par la mère : la trajectoire «fonctionnaire » est marquée par une part importante de mères travaillant dans le secteur public (31\% contre $28 \%$ pour l'effectif total). Toutefois, ce constat est à relativiser dans la mesure où les mères des jeunes s'insérant en CDI sont aussi plus souvent dans le secteur public que pour l'échantillon global. Si l'on élargit cette notion de transmission au secteur privé, les jeunes des trajectoires à dominante « privée » ont plus souvent une mère travaillant dans le secteur privé que le reste de l'échantillon. À l'inverse, la mère est plus souvent inactive que dans l'échantillon global pour les trajectoires à dominante « instable » (sauf « CDD privé instable » pour lesquels la mère est essentiellement en emploi dans le secteur privé).

Globalement, l'hypothèse de transmission d'un statut est néanmoins difficile à prendre en compte sur la base des simples statistiques descriptives et ceci d'autant plus que les jeunes passés uniquement par le secteur privé sont exclus de l'analyse. On y portera une attention particulière dans l'analyse économétrique qui suit.

Les secteurs d'emploi sur le marché du travail des conjoints apportent des éléments d'information permettant de caractériser les jeunes pas- 
sant par la fonction publique. On observe globalement un effet de proximité des situations professionnelles entre conjoints : les jeunes de la trajectoire «fonctionnaire » ont plus fréquemment que la moyenne un conjoint travaillant dans le secteur public (39\% contre $31 \%$ ), pour leur part, les jeunes en trajectoire à dominante «privée » («CDD privé insérant», «CDI privé ») ont davantage que la moyenne un conjoint dans le secteur privé (resp. $63 \%$ et $59 \%$ contre $51 \%$ ), et enfin, les jeunes en trajectoires à dominante «instable » (« inactifs », « chômage ») ont plus souvent un conjoint inactif (resp. $28 \%$ et $23 \%$ contre $18 \%$ en moyenne). Mais s'intéresser au secteur d'emploi du conjoint n'a qu'un caractère informatif s'il n'est pas rattaché à la fois à la date de mise en couple (avant ou après l'entrée dans l'emploi public en particulier) et au genre du conjoint. Les fonctionnaires n'ont ainsi pas plus souvent un conjoint inactif que l'ensemble de la population, sachant que les fonctionnaires sont majoritairement des femmes.

\section{Trajectoire et formation}

Les structures des niveaux de formation se différencient nettement entre trajectoires. Les jeunes de la trajectoire «fonctionnaire » sont largement plus formés que la moyenne : ils sont plus fréquemment de niveau II de formation initiale (54\% contre seulement $30 \%$ pour l'ensemble de l'effectif). $75 \%$ de l'effectif de cette trajectoire (54\% pour l'ensemble de l'échantillon) ont un diplôme supérieur ou équivalent à la licence (niveau III et plus). Ce constat va dans le sens de ce qui est généralement observé sur la population des fonctionnaires : «près d'un diplômé sur deux, titulaire d'au moins une licence ou d'un diplôme équivalent entre dans la fonction publique » (Audier, 1997, à partir de l'Enquête Emploi).

En revanche, appartiennent à cette trajectoire «fonctionnaire » des jeunes de niveau de formation initiale inférieur au niveau III en proportion moindre que dans l'échantillon global. En particulier, les jeunes entamant un cursus universitaire après leur bac et s'arrêtant avant d'en être diplômés (niveau IV +) entrent plus fréquemment dans la fonction publique. Ces derniers, absents de la trajectoire d'insertion directe dans la fonction publique, appartiennent à celle des «emplois jeunes » et à celle des « inactifs » (qui transitent surtout vers un emploi de fonctionnaire).

Plus généralement, les niveaux de qualification sont moins élevés dans les trajectoires à domi- nante «privée », relativement à l'ensemble de l'échantillon; au contraire, les qualifications sont plus élevées que la moyenne de l'échantillon dans les trajectoires à dominante « publique » et ce sans égard à la stabilité de la trajectoire : les trajectoires privées stables concernent des niveaux de qualification moins élevés que la moyenne, les trajectoires publiques instables ont, au contraire, un niveau de qualification plus élevé que la moyenne. La trajectoire caractérisée par les niveaux de qualification les plus bas est logiquement celle du « chômage ».

\section{Trajectoire et situation finale}

Les situations en mars 2001 ont permis de caractériser les différentes trajectoires types. On a donc globalement une correspondance entre l'état final sur le marché du travail et l'intitulé de la trajectoire. Il est alors intéressant de caractériser les rapports entre des trajectoires instables et l'emploi final stable (cf. annexe 4, tableau B). Il s'agit en premier lieu de la trajectoire « inactif» où les jeunes sont pour $42 \%$ fonctionnaires en mars 2001, et pour qui l'insertion dans la fonction publique a été différée. En second lieu, les jeunes de la trajectoire «CDD privé insérant » se retrouvent à $25 \%$ dans un emploi de fonctionnaire : ainsi, dans nombre de cas, c'est le passage par un emploi du secteur privé qui va finalement aboutir à une insertion dans la fonction publique en tant que titulaire.

Les individus de la trajectoire « fonctionnaire », qui sont aussi les plus qualifiés, occupent en mars 2001 plus que la moyenne des emplois de cadres. Au contraire, l'insertion des personnes de l'échantillon dans le secteur privé, représentée par la trajectoire «CDI privé », est caractérisée par des emplois d'ouvriers et d'employés. De même, dans les autres trajectoires «privées» («CDD privé instable», « CDD privé insérant») les jeunes occupent, en mars 2001, des emplois d'ouvriers ou d'employés plus fréquemment que la moyenne. Ainsi, les jeunes passés au moins une fois par l'emploi public temporaire, lorsqu'ils sont en emploi du secteur privé trois ans après la sortie de formation initiale, le sont sur des niveaux d'emploi moins élevés que les jeunes qui sont dans le secteur public à la même échéance.

Le salaire médian d'embauche dans le dernier emploi occupé dans la trajectoire des fonctionnaires est le plus élevé de l'ensemble des parcours types. Avec un salaire d'embauche également élevé, la trajectoire dite des « inactifs » est 
telle que la diminution de l'inactivité (ou formation, ou reprise d'études) se fait au profit du statut de fonctionnaire : ainsi, les deux niveaux de salaire médian les plus élevés à l'embauche renvoient à des emplois de fonctionnaire. Par ailleurs, ce salaire est aussi celui qui progresse le plus, de 180 euros en deux ans (pour une ancienneté dans le dernier emploi de 25 mois). Rappelons que ces niveaux de salaire dépendent du niveau de formation des jeunes, les plus élevés dans la trajectoire «fonctionnaire », ainsi que des catégories socioprofessionnelles occupées (35\% d'entre eux sont cadres).

La trajectoire «fonctionnaire » est constituée, dans plus de la moitié des cas, de fonctionnaires de l'Éducation nationale à la date d'enquête. Les jeunes de la trajectoire «emplois jeunes » se répartissent entre les emplois de l'Éducation nationale (50\% des cas) et les emplois de la fonction publique territoriale (31\%). S'agissant du parcours «CDD privé insérant», l'emploi s'exerce plus souvent dans le secteur public trois ans après la fin des études (29\% seulement se situent dans le secteur privé).

\section{Fonction publique d'État et fonction publique territoriale}

Les différents modes de recours aux contrats de travail dans les deux fonctions publiques incitent, pour chacune des trajectoires établies, à considérer la part des emplois occupés dans la fonction publique territoriale en mars 2001. Cette part est la plus notable au sein des trajectoires dont l'emploi final est non pérenne.

Pour la trajectoire «fonctionnaires », $17 \%$ des emplois s'exercent dans la fonction publique territoriale contre $80 \%$ au sein de la fonction publique d'État. L'importance du poids des emplois dans la fonction publique territoriale en fin de période d'observation dans la trajectoire « chômage » peut trouver deux explications. La première tient au niveau de formation moins élevé des jeunes dans cette fonction publique et donc à leur risque accru de chômage. La seconde tient au mode de recrutement spécifique de la fonction publique territoriale : contrairement aux procédures de la fonction publique d'État, la réussite à un concours de la fonction publique territoriale ne donne pas accès à un poste mais à une liste d'aptitude autorisant à se porter candidat sur des emplois vacants de la fonction publique territoriale. Il peut donc y avoir un temps de chômage entre une réussite à un concours et l'occupation d'un emploi de la fonction publique territoriale. Enfin, de façon attendue, la situation finale dans les trajectoires « emplois jeunes », « CDD public » et « CDD public instable » correspond fréquemment à un emploi dans la fonction publique territoriale, cette dernière privilégiant en effet le recours aux contrats emplois jeunes et à durée déterminée (Dauty et Lemistre, 2003).

\section{Les déterminants individuels de l'appartenance aux différentes trajectoires types}

L'objet est ici d'identifier parmi les caractéristiques individuelles sur le marché du travail les déterminants de l'appartenance aux parcours types précédemment établis. Dans cette perspective, on estime un modèle logit multinomial (Gourriéroux, 1984) dans lequel la typologie (9 modalités) est la variable à expliquer et où les caractéristiques individuelles constituent les facteurs explicatifs. La modalité de référence est l'appartenance à la trajectoire type «fonctionnaire ».

Les résultats d'estimation permettent d'établir qu'être un homme explique l'appartenance à des parcours types différents de ceux des femmes (cf. annexe 5). Les hommes ont une moins grande probabilité d'appartenir à des trajectoires dont l'état dominant est le CDD privé, l'emploi jeune ou le chômage qu'à la trajectoire «fonctionnaire» (parcours type de référence). À l'inverse, ils montrent des propensions plus grandes que les femmes d'appartenir à la trajectoire des inactifs dont l'issue majeure est le statut de fonctionnaire, relativement à la trajectoire de référence (accès direct à ce statut). De même, leur probabilité de se situer dans le parcours «CDD public instable» est relativement plus grande : ce dernier statut, détenu en fin de période d'observation, semble s'apparenter à un pis-aller plus qu'à un véritable choix.

Concernant l'effet du niveau de formation initiale, être diplômé de niveau II agit positivement sur la probabilité d'appartenir au parcours «fonctionnaire ». Tous les autres niveaux inférieurs au niveau II expliquent l'appartenance à des trajectoires autres que cette dernière. Être sortant de l'enseignement supérieur sans en être diplômé (niveau IV +) favorise l'appartenance à la trajectoire « emploi jeune » relativement au parcours de référence.

Résider en province plutôt qu'à Paris ou dans sa région en fin de formation initiale favorise la 
participation aux parcours plus instables et pénalise l'entrée dans le parcours «CDI privé » comparativement au parcours de référence.

Vivre en couple accroît les chances d'appartenir à la trajectoire «CDI privé » relativement à celles des fonctionnaires et réduit la probabilité de se situer dans des trajectoires instables. Lorsque le conjoint travaille dans le secteur public, la probabilité de faire partie d'une trajectoire autre que «fonctionnaire » diminue. De même, le fait que le père ou la mère travaille dans ce secteur accroît la probabilité d'appartenir à la trajectoire de référence, sans que cet effet soit pour autant déterminant.

Ces résultats tendent à montrer que le rôle de l'emploi public dans l'insertion des jeunes diffère particulièrement selon le sexe et le niveau de formation. Les femmes et les plus diplômés s'insèrent plus rapidement et plus fréquemment dans le secteur public contrairement aux hommes et aux moins diplômés de la population étudiée.

\section{Le rôle des CDD dans l'accès à un emploi pérenne}

$\mathbf{L}$ a construction de trajectoires types puis l'analyse des caractéristiques expliquant les probabilités d'appartenir à l'une ou l'autre de ces trajectoires ont apporté des premiers éléments de réponse sur le rôle joué par l'emploi public dans l'insertion des jeunes. Il convient cependant, à ce stade, de s'interroger plus explicitement sur l'efficacité des emplois publics autres que les emplois de fonctionnaire sur l'insertion des jeunes et en particulier sur leur accès à un statut pérenne au terme des trois années d'observation. En effet, posséder une expérience professionnelle préalable dans le secteur public favorise-t-il l'accès à un emploi de fonctionnaire ou en CDI dans le secteur privé ? Autrement dit, dès lors qu'une telle expérience professionnelle facilite l'accès à une situation d'emploi stable après trois ans de vie active, on peut supposer que l'emploi public en CDD est considéré comme une accumulation d'expérience professionnelle valorisable. Par ailleurs, dans quelle mesure, contrairement au CDD du secteur privé, le CDD du secteur public améliore-t-il les chances d'accès au marché interne de la fonction publique et à l'inverse, freine-t-il l'accès au CDI du secteur privé ?

\section{Secteur public versus secteur privé : une expérience passée déterminante sur l'accès à une situation pérenne}

Une telle évaluation suppose de travailler sur l'ensemble de la population et non plus exclusivement sur les jeunes passés par un emploi public. Au-delà, une analyse de ce type confronte l'économètre à la présence classique d'endogénéité. En effet, les jeunes qui passent par l'emploi public ont des caractéristiques spécifiques qui les distinguent de l'ensemble des jeunes (cf. supra). De la même façon, les facteurs explicatifs observés ou non du passage par un CDD du secteur privé peuvent également être des déterminants de l'accès au statut de fonctionnaire ou à un CDI. Si la probabilité de passage par un CDD de l'un et l'autre des secteurs n'est pas estimée, le rôle propre des caractéristiques de l'individu ne peut être distingué de celui des caractéristiques de l'emploi en CDD. Par exemple, les difficultés de certains jeunes à se stabiliser sur le marché du travail peuvent davantage relever de leurs attributs individuels déterminant leur passage par un CDD public plutôt que de cette expérience professionnelle elle-même. Tenir compte du caractère endogène du passage par un CDD public ou privé conduit à estimer simultanément, d'une part les probabilités d'avoir occupé un CDD du secteur public, d'avoir occupé un CDD du secteur privé et d'être fonctionnaire en mars 2001 ; d'autre part, les probabilités d'avoir occupé un CDD du secteur public, d'avoir occupé un CDD du secteur privé et d'être en CDI en mars 2001. À cet effet, on recourt à l'estimation de deux modèles probit trivarié, le premier concernant l'obtention du statut de fonctionnaire, le second portant sur l'accès au CDI (cf. encadré 3).

L'interrogation portant sur la valorisation potentielle des expériences antérieures acquises dans les secteurs public et privé, on ne retient que les séquences d'emploi en CDD dont la durée est de 6 mois au moins. Si le rôle des emplois aidés public sur l'accès à un statut pérenne mérite qu'on s'y intéresse, il s'avère que pour la population d'ensemble, ces emplois aidés sont massivement des emplois jeunes $(89 \%)$ dont l'issue n'est que trop rarement observée pour pouvoir en tirer des conclusions.

À la date d'enquête, $44,5 \%$ des individus détiennent un CDI parmi lesquels $27 \%$ sont passés par un CDD du secteur privé de plus de 6 mois et $1,5 \%$ ont connu ce même type de contrat mais dans le secteur public. Environ $8 \%$ des 
jeunes ont le statut de fonctionnaire en mars 2001. Le passage antérieur par un emploi instable du secteur privé est largement moins fréquent que pour les jeunes en CDI. En effet, ils ne sont plus que $8 \%$ dans ce cas alors qu'un sur quatre a connu un CDD de plus de 6 mois dans le secteur public.

L'hypothèse d'absence de corrélation entre les variables inobservées des trois équations est rejetée par un test du ratio de vraisemblance (cf. tableau de résultats annexe 6), confirmant la pertinence de la modélisation par des modèles probit trivariés. Il s'avère que les variables inobservées déterminant la probabilité du passage par un CDD de plus de 6 mois du secteur public et du secteur privé ne sont pas significativement corrélées avec les variables inobservées expliquant l'accès au statut de fonctionnaire. En revanche, il existe des caractéristiques non observées déterminant le passage par un
CDD du secteur public - de plus de 6 mois corrélées négativement avec les attributs non observés explicatifs du passage par un CDD privé de plus de 6 mois. Quant aux facteurs inobservés déterminant la probabilité d'être en CDI à la date d'enquête, ils sont corrélés négativement avec ceux agissant sur la probabilité du passage par un CDD du secteur public, la corrélation devenant positive lorsqu'il s'agit d'un CDD du secteur privé. Parmi ces variables inobservées pourrait figurer l'aversion pour le risque de chômage qui serait plus élevée chez les futurs salariés du secteur public qu'au sein du secteur privé (Fougère et Pouget, 2003). Cet élément explicatif pourrait, par exemple, justifier la corrélation négative entre la probabilité d'avoir expérimenté un CDD du secteur public et la probabilité d'accès final à un CDI.

Avant même l'accès aux statuts pérennes des secteurs public et privé, les déterminants des pas-

Encadré 3

\section{LES MODÈLES PROBIT TRIVARIÉS}

Afin de contrôler les problèmes d'endogénéité, un système de variables qualitatives dépendantes décrivant le passage par un CDD du secteur public et du secteur privé ainsi que l'accès au statut de fonctionnaire (ou à un $\mathrm{CDI}$ ) à la date d'enquête est estimé par des modèles de forme probit. Les passages par un CDD dans les deux secteurs d'activité au cours des trois premières années de vie active interviennent comme variables explicatives de l'accès à cette dernière situation. Formellement, les trois processus en cause, reliés entre eux, sont modélisés à partir de variables latentes $y_{s i}^{*}$, $s=1,2$ ou 3 , de la façon suivante :

$$
\begin{aligned}
& y_{1 i}=\left\{\begin{array}{lll}
1 & \text { si } & y_{1 i}^{*}>0 \\
0 & \text { si } & y_{1 i}^{*} \leq 0
\end{array} \text { où } \quad y_{1 i}^{*}=X_{1 i}^{\prime} \beta_{1}+\varepsilon_{1 i}\right. \\
& y_{2 i}=\left\{\begin{array}{lll}
1 & \text { si } & y_{2 i}^{*}>0 \\
0 & \text { si } & y_{2 i}^{*} \leq 0
\end{array} \quad \text { où } \quad y_{2 i}^{*}=X_{2 i}{ }^{\prime} \beta_{2}+\varepsilon_{2 i}\right. \\
& y_{3 i}=\left\{\begin{array}{lll}
1 & \text { si } & y_{3 i}^{*}>0 \\
0 & \text { si } & y_{3 i}^{*} \leq 0
\end{array} \text { où } \quad y_{3 i}^{*}=X_{3 i}^{\prime} \beta_{3}+\gamma_{1} y_{1 i}+\right. \\
& \gamma_{2} y_{2 i}+\varepsilon_{3 i}
\end{aligned}
$$

La variable dichotomique $y_{1}$ correspond au passage par un CDD du secteur public de plus de 6 mois avant mars 2001, $y_{2}$ au passage par un CDD du secteur privé de plus de 6 mois avant cette date. Enfin, $y_{3}$ prend la valeur 1 si l'individu est fonctionnaire (respectivement, a un $\mathrm{CDI}$ ) à la date d'enquête, 0 sinon. Cette variable est observée après les variables $y_{1}$ et $y_{2}$. Ces dernières figurant comme explicatives de $y_{3}$, l'estimation du modèle requiert que certaines explicatives des deux premières équations ne soient pas incluses dans la troisième équation. Le pays de naissance du père est la variable que l'on a choisi d'écarter dans la mesure où son effet sur l'accès au statut de fonctionnaire s'est avéré non significatif, le pays de naissance du jeune étant retenu.

Une spécification de type probit repose sur l'hypothèse de normalité de la loi des composantes d'erreur des modèles de régression sous-jacents, la normalisation à 1 des variances étant nécessaire pour des raisons d'identification. On a :

$$
\left(\begin{array}{l}
\varepsilon_{1} \\
\varepsilon_{2} \\
\varepsilon_{3}
\end{array}\right) \rightarrow N(0, \Sigma) \text { et } \Sigma=\left(\begin{array}{ccc}
1 & \rho_{12} & \rho_{13} \\
\rho_{12} & 1 & \rho_{23} \\
\rho_{13} & \rho_{23} & 1
\end{array}\right)
$$

Pour l'écriture de la fonction de vraisemblance et son estimation, voir Greene (2003) et l'extension à la programmation de Stata proposée par Terracol (2002) dans le cas présent où les variables $y_{1}$ et $y_{2}$ sont endogènes.

Dans la mesure où l'objet est d'évaluer l'effet causal du passage éventuel par un emploi instable dans le secteur privé et/ou dans le secteur public sur l'accès à chacun des deux types d'emplois pérennes, un système de quatre équations, un modèle probit quadrivarié, aurait été la modélisation idéale. Cependant, compte tenu de la complexité des calculs d'intégration que l'estimation d'un tel modèle suppose (Greene, 2003, pp. 7-14), les techniques d'estimation actuelles conduisent à l'estimation de deux modèles probit trivariés. 
sages par des emplois précaires dans l'un et l'autre de ces secteurs se différencient, en particulier pour ce qui concerne les liens de l'entourage familial (père, mère) avec le secteur public (cf. annexe 6). Avoir une mère salariée de la fonction publique prédispose à une expérience professionnelle dans ce secteur (Audier, 2000). À l'inverse, l'appartenance des parents au salariat privé à la fin des études augmente la probabilité de passage par un CDD du secteur privé. Si les spécialités industrielles de formation initiale permettent le passage par un CDD privé, davantage que les spécialités tertiaires et générales, ce sont ces dernières qui favorisent l'emploi en CDD public, plus encore que les spécialités tertiaires. Enfin, être né en France favorise le passage par un CDD de plus de 6 mois dans l'un comme dans l'autre des secteurs, davantage encore dans le secteur public. Avoir un père né à l'étranger limite le passage par un CDD.

Les déterminants de l'accès de la cohorte 1998 au statut de fonctionnaire ou au CDI du privé se révèlent être différents (cf. annexe 6). L'analyse des niveaux de formation initiale montre que les chances d'accès à ces deux situations pérennes diffèrent avec le niveau des études : si la fonction publique recrute plus spécifiquement au niveau II de formation, en revanche, la probabilité d'être en CDI trois ans plus tard est d'autant plus grande que le niveau de diplôme est élevé. La spécialité de formation tertiaire plutôt qu'industrielle ou générale permet un meilleur accès au statut de fonctionnaire, toutes choses égales par ailleurs. L'obtention d'un CDI est, quant à elle, davantage favorisée par les spécialités de formation industrielles. À la fin des études, habiter la province réduit la probabilité d'être en CDI trois ans plus tard. À l'inverse, l'accès au statut de fonctionnaire est plus fréquent lorsque les jeunes habitent la province ou en Île-de-France, plutôt qu'à Paris en 1998.

Le sexe est également une variable déterminante du statut d'emploi en mars 2001. Si être un homme accroît les chances d'être en CDI, cela diminue celles d'être fonctionnaire. Ce résultat est conforme à la féminisation connue de la fonction publique (Audier, 2000) et à l'attrait de la main-d'œuvre féminine pour ce statut comme l'a montré l'analyse des probabilités d'appartenance aux différentes trajectoires. La proximité familiale avec la fonction publique (avoir un conjoint, un père et/ou une mère salarié dans le secteur public) améliore les chances d'être fonctionnaire en mars 2001 et au contraire les réduit quand il s'agit de l'obtention d'un CDI dans le secteur privé. Le lieu de nais- sance n'a aucun impact significatif sur l'accès à un statut pérenne en mars 2001.

Concernant le parcours professionnel antérieur, le nombre des épisodes de chômage au cours des premières années de vie active est pénalisant pour l'accès au statut de fonctionnaire (cf. annexe 6). L'introduction au sein des variables explicatives de la durée d'expérience professionnelle sur les trois années de vie active, rend non significatif l'effet de la récurrence des épisodes de chômage pour l'accès à un CDI en mars 2001. Ce résultat met en avant que l'expérience professionnelle compense l'effet négatif du passage par le chômage pour l'accès à un CDI, alors que ceci n'est pas vrai dans le cas de l'accès au statut de fonctionnaire. Quant aux mobilités professionnelles (changement d'établissement employeur), elles ont un effet positif sur l'accès au statut de fonctionnaire et négatif pour l'accès à un CDI. Ainsi, toutes choses égales par ailleurs, la multiplicité des expériences sur le marché du travail, et leur durée totale, favorisent l'accès au statut de fonctionnaire.

Enfin, si on s'intéresse au rôle des emplois précaires dans l'accès aux emplois stables, on observe que l'importance des CDD du secteur public dans l'accès à un statut pérenne dans ce secteur est inverse à celle qu'il tient dans l'accès à un CDI. En effet, le passage par un CDD de plus de 6 mois dans le secteur public accroît les chances de devenir fonctionnaire en mars 2001 et réduit celles d'accéder à un CDI. Si le passage par un emploi en CDD du secteur privé - de plus de 6 mois - ne modifie pas significativement les chances d'accès à un emploi en CDI, il diminue en revanche celles de devenir fonctionnaire, plutôt qu'occuper un tout autre statut (y compris un CDI). Au vu de ces résultats, il semble donc que l'emploi public en CDD soit un tremplin pour devenir fonctionnaire et qu'en revanche il n'augmente pas les chances d'obtenir un CDI dans le secteur privé.

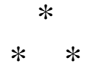

Pour aller plus loin sur le rôle de l'emploi public dans l'insertion des jeunes, une piste de recherche consisterait à prendre en compte la séquentialité des états connus sur le marché du travail. L'idée est d'évaluer l'impact d'un épisode de chômage comparativement à un épisode

8. Cette dernière caractéristique est la variable que l'on a choisi de ne pas retenir comme explicative dans les équations d'accès au statut de fonctionnaire et à un $\mathrm{CDI}$ dans la mesure où le pays de naissance du jeune a lui-même été introduit. 
d'emploi public précaire, par exemple, sur l'issue (observée) de la trajectoire. Une telle question nécessiterait d'estimer un modèle de durée à risques concurrents afin de cerner la probabilité de transiter vers le statut de fonctionnaire ou le CDI selon le parcours antérieur, mais aussi un modèle de transition, forme économétrique la plus adaptée pour étudier l'impact de différents états connus sur le marché du travail dans l'évolution de la trajectoire.

Les auteurs remercient Éric Cahuzac, Arnaud Dupray, Jean-François Giret, Olivier Joseph, Stéphane Luchini et Michel Théry pour leurs conseils, ainsi que les deux rapporteurs anonymes de la revue.

Ce travail s'inscrit dans le cadre d'un projet de recherche commandité par le Ministère de la Recherche sur le thème du travail dans la fonction publique : "Les déterminants des entrées dans les fonctions publiques : parcours du combattant ou pis-aller?». L'équipe de recherche, sous la responsabilité de Florence Audier (Matisse), comprend, outre les auteurs, Dominique Meurs (Ermes), Marion Lambert et Coralie Perez (Céreq).

\section{BIBLIOGRAPHIE}

Audier F. (2000), « La transmission du statut dans la fonction publique », Économie et Statistique, $n^{\circ} 337-338$, pp. 121-133.

Audier F. (1997), « La fonction publique, un débouché majeur pour les mieux formés », Économie et Statistique, $\mathrm{n}^{\circ}$ 304-305, pp. 137-148.

Béduwé C. et Cahuzac E. (1997), «Première expérience professionnelle avant le diplôme. Quelle insertion pour les étudiants de second cycle universitaire ? », Formation Emploi, n 58 , Céreq, La documentation Française, Paris.

Béduwé C., Dauty F. et Espinasse J.-M. (1995), «Trajectoires-type d'insertion professionnelle », note LIRHE (190(95-11), 2ème Journées d'études sur les données longitudinales sur le marché du travail, Caen, Document Séminaires du Céreq, $\mathrm{n}^{\circ} 112$.

Brenot-Ouldali A. (1999), «Les salariés du secteur public. Les fonctionnaires et les autres », Futuribles, ${ }^{\circ}$ 242, mai, pp. 55-58.

Dauty F. et Lemistre P. (2003), « La fonction publique territoriale : un employeur spécifique », Xèmes Journées d'Études sur les données longitudinales sur le marché du travail, Caen, Documents Séminaires du Céreq, n 171, pp. 299-310.

Epiphane D., Giret J.-F., Hallier P., Lopez A. et Sigot J.-C. (2001), « Génération 98 - À qui a profité l'embellie économique? », Bref, $\mathrm{n}^{\circ} 181$, décembre, Céreq.

Espinasse J.-M. (1994), « Enquête de cheminement, chronogrammes et classification automatique », 1 ères Journées d'Études sur les données longitudinales sur le marché du travail, Toulouse, Documents Séminaires du Céreq, $\mathrm{n}^{\circ}$ 99, pp. 193-218.
Espinasse J.-M. et Giret J.-F. (1996), «Chronogrammes et trajectoires. Un premier bilan », IIIÌmes Journées d'Études sur les données longitudinales sur le marché du travail, Rennes, Documents Séminaires du Céreq, n 115, pp. 333-346.

Fougère D. et Pouget J. (2003), « Les déterminants économiques de l'entrée dans la fonction publique », ce numéro.

Gouriéroux C. (1984), Économétrie des variables qualitatives, Économica.

Greene W.H. (2003), Econometric Analysis, $5^{\text {e }}$ édition, Prentice Hall, New York University.

Guillotin Y.et Meurs D. (1999), Wage Heterogeneity in the French Public Sector: Some First Insights, in Public Sector Pay Determination in the European Union, Macmillan.

Observatoire de l'emploi public (2001), Rapport annuel, Ministère de la fonction publique et de la réforme de l'État, juin.

di Paola V. (2000), L'hétérogénéité non observée dans les modèles de durée - Une application au marché du travail des jeunes, Thèse pour le doctorat en sciences économiques, Université de la Méditerranée, janvier 2000, 196 p.

de Singly F. et Thélot C. (1988), Gens du public, gens du privé. La grande différence, Dunod.

Terracol A. (2002), «Triprobit and the GHK Simulator: A Short Note », Annexe à la commande Stata Triprobit. 
ANNEXE 1

Tableau A

Caractéristiques individuelles

\begin{tabular}{|c|c|c|}
\hline Variables & Échantillon " secteur public » & Échantillon total \\
\hline Homme & 40,6 & 51,2 \\
\hline \multicolumn{3}{|l|}{ Niveau de formation (1) } \\
\hline Niveau I & 7,9 & 7,8 \\
\hline Niveau II & 29,8 & 10,9 \\
\hline Niveau III & 16,3 & 18,6 \\
\hline Niveau IV & 11,7 & 17,4 \\
\hline Niveau IV + & 17,1 & 12,3 \\
\hline Niveau V & 13,9 & 25,3 \\
\hline Niveau Vbis & 2,2 & 4,4 \\
\hline Niveau VI & 1,2 & 3,5 \\
\hline \multicolumn{3}{|l|}{ Spécialité de formation } \\
\hline Générale & 31,6 & 19,4 \\
\hline Industrielle & 18,6 & 33,9 \\
\hline Tertiaire & 49,8 & 46,7 \\
\hline Né(e) en France & 97,2 & 95,8 \\
\hline Vie en couple & 41,6 & 36,6 \\
\hline Avoir un enfant & 12,2 & 12,5 \\
\hline \multicolumn{3}{|l|}{ Situation du conjoint } \\
\hline Emploi public & 30,7 & 21,5 \\
\hline Emploi privé & 51,4 & 58,1 \\
\hline Inactif & 17,9 & 20,4 \\
\hline \multicolumn{3}{|l|}{ Situation du père (fin étude) } \\
\hline Emploi public & 27,0 & 23,3 \\
\hline Emploi privé & 49,1 & 54,4 \\
\hline Inactif & 23,8 & 22,3 \\
\hline \multicolumn{3}{|l|}{ Situation de la mère (fin étude) } \\
\hline Emploi public & 27,9 & 22,81 \\
\hline Emploi privé & 32,9 & 36,4 \\
\hline Inactif & 39,2 & 40,7 \\
\hline \multicolumn{3}{|l|}{ Pays de naissance du père } \\
\hline France & 85,3 & 83,4 \\
\hline Afrique & 9,7 & 10,3 \\
\hline Autres & 5,0 & 6,3 \\
\hline Âge médian en 1998 & 22 & 21 \\
\hline Effectifs (pondérés) & 112121 & 741537 \\
\hline \multicolumn{3}{|c|}{$\begin{array}{l}\text { 1. Niveau VI : sortie avant la troisième, Niveau Vbis : sorties de troisième et de second cycle court avant l'année terminale, } \\
\text { Niveau V : sorties de l'année terminale de second cycle court professionnel et abandon de la scolarité du second cycle long avant la } \\
\text { terminale, Niveau IV : sorties des classes terminales du second cycle long (niveau IV secondaire) et abandon des scolarisations post- } \\
\text { baccalauréat avant d'atteindre le niveau III (niveau IV supérieur), Niveau III : sorties avec un diplôme de niveau bac }+2 \text { ans, DUT, } \\
\text { DEUG, BTS, Diplôme para-médical, Niveaux I et II : sorties avec un diplôme de deuxième ou de troisième cycle universitaire ou un } \\
\text { diplôme de grande école. }\end{array}$} \\
\hline
\end{tabular}

Lecture : parmi les individus passés au moins une fois par un emploi public, 40,6\% sont des hommes, alors que parmi la population des jeunes sortis de formation initiale en 1998, cette part est de 51,17\%.

Source : Génération 98. 
Tableau B

Parcours sur le marché du travail

\begin{tabular}{|c|c|c|}
\hline Variables & Échantillon « secteur public » & Échantillon total \\
\hline \multicolumn{3}{|l|}{ Nombre de séquences de chômage } \\
\hline 0 & 55,8 & 54,1 \\
\hline 1 & 28,6 & 31,5 \\
\hline 2 & 9,7 & 9,7 \\
\hline 3 et plus & 5,9 & 4,7 \\
\hline Nombre de séquences d'emploi & 0,0 & 5,7 \\
\hline 1 & 36,3 & 40,4 \\
\hline 2 & 34,7 & 30,7 \\
\hline 3 & 16,9 & 13,8 \\
\hline 4 et plus & 12,1 & 9,4 \\
\hline Salaire médian en mars 2001 (en euros) & 1070 & 1100 \\
\hline Salaire médian à l'embauche (1) (en euros) & 990 & 990 \\
\hline Ancienneté dans le dernier emploi (en mois) & 17 & 17 \\
\hline \multicolumn{3}{|l|}{ Situation en mars 2001} \\
\hline Fonctionnaire & 35,2 & 7,8 \\
\hline CDI du privé & 9,7 & 44,4 \\
\hline CDD du privé (2) & 6,5 & 15,8 \\
\hline CDD du public (3) & 17,9 & 4,3 \\
\hline Emplois aidés du secteur public & 19,3 & 3,8 \\
\hline Emplois aidés du secteur privé & 2,1 & 3,7 \\
\hline Chômage & 4,8 & 9,2 \\
\hline Inactivité-Formation & 4,3 & 8,4 \\
\hline Travailleurs indépendants & - & 2,5 \\
\hline \multicolumn{3}{|l|}{ Secteur d'emploi en mars 2001} \\
\hline Éducation nationale & 34,3 & 6,0 \\
\hline Armées & 7,6 & 1,3 \\
\hline Autres ministères & 16,6 & 3,0 \\
\hline Collectivités territoriales & 21,1 & 3,7 \\
\hline Para-public & 1,4 & 9,3 \\
\hline Secteur privé & 19,1 & 76,6 \\
\hline Effectifs & 112121 & 741537 \\
\hline \multicolumn{3}{|c|}{$\begin{array}{l}\text { 1. Salaire d'embauche de la séquence d'emploi à la date d'enquête (mars 2001). } \\
\text { 2. Y compris l'emploi intérimaire. } \\
\text { 3. Y compris l'emploi intérimaire. }\end{array}$} \\
\hline
\end{tabular}

Lecture : parmi les individus passés au moins une fois par un emploi public, 55,8 \% n'ont connu aucun épisode de chômage, parmi la population des jeunes sortis de formation initiale en 1998, cette part est de $54 \%$.

Source : Génération 98 
ANNEXE 2

Modèle Probit à variable dépendante "Avoir connu au moins un épisode d'emploi public "

\begin{tabular}{|c|c|c|}
\hline Variables & Paramètres & P-value \\
\hline Constante & $-2,936$ & 0,0000 \\
\hline Homme & $-0,2485$ & 0,0000 \\
\hline Âge en 1998 & 0,0617 & 0,0000 \\
\hline \multicolumn{3}{|l|}{ Niveau de formation } \\
\hline Niveau I & Réf. & \\
\hline Niveau II & 0,5829 & 0,0000 \\
\hline Niveau III & $-0,2008$ & 0,0000 \\
\hline Niveau IV & $-0,3513$ & 0,0000 \\
\hline Niveau IV+ & 0,0868 & 0,0049 \\
\hline Niveau V & $-0,3472$ & 0,0000 \\
\hline Niveau Vbis & $-0,2508$ & 0,0000 \\
\hline Niveau VI & $-0,2077$ & 0,0040 \\
\hline Nombre de séquences de chômage & $-0,0213$ & 0,0104 \\
\hline Nombre de séquences d'emploi & 0,1124 & 0,0000 \\
\hline \multicolumn{3}{|l|}{ Lieu de résidence en fin d'études } \\
\hline Paris & Réf. & \\
\hline Île-de-France & 0,02949 & 0,0000 \\
\hline Province & 0,2442 & 0,0000 \\
\hline Vie en couple & $-0,1823$ & 0,0000 \\
\hline Avoir un enfant & $-0,0135$ & 0,5690 \\
\hline \multicolumn{3}{|l|}{ Lieu de résidence en mars 2001} \\
\hline Paris & Réf. & \\
\hline Île-de-France & 0,3212 & 0,0000 \\
\hline Province & 0,2949 & 0,0000 \\
\hline \multicolumn{3}{|l|}{ Situation du conjoint en mars 2001} \\
\hline Secteur privé & Réf. & \\
\hline Inactif & 0,1648 & 0,0000 \\
\hline Secteur public & 0,3995 & 0,0000 \\
\hline \multicolumn{3}{|l|}{ Situation du père en 1998} \\
\hline Secteur privé & Réf. & \\
\hline Inactif & 0,1170 & 0,0000 \\
\hline Secteur public & 0,1802 & 0,0000 \\
\hline \multicolumn{3}{|l|}{ Situation de la mère en 1998} \\
\hline Secteur privé & Réf. & \\
\hline Inactive & 0,0897 & 0,0000 \\
\hline Secteur public & 0,1858 & 0,0000 \\
\hline \multicolumn{3}{|l|}{ Pays de naissance } \\
\hline France & Réf. & \\
\hline Autres & $-0,2127$ & 0,0000 \\
\hline \multicolumn{3}{|l|}{ Pays de naissance du père } \\
\hline France & Réf. & \\
\hline Autres & $-0,0405$ & 0,0594 \\
\hline Log-vraisemblance & \multicolumn{2}{|c|}{$-40358,69$} \\
\hline
\end{tabular}

Champ : échantillon total.

Source : Génération 98. 


\section{LES TRAJECTOIRES TYPES}

Tableau

Effectifs par trajectoire type

\begin{tabular}{|l|r|r|r|r|r|r|r|r|r|r|}
\hline & \multicolumn{10}{|c|}{ Trajectoires (1) } \\
\cline { 2 - 14 } & 1 & 2 & 3 & 4 & 5 & 6 & 7 & 8 & 9 & Total \\
\hline Effectifs (pondéré) & 27367 & 7133 & 17758 & 11420 & 13883 & 7112 & 8337 & 10686 & 8425 & 112121 \\
\hline Effectifs & 2612 & 561 & 1253 & 859 & 1140 & 484 & 646 & 785 & 607 & 8947 \\
\hline 1. Se reporter au tableau 1 pour la liste des trajectoires.
\end{tabular}

Lecture : parmi les individus passés au moins une fois par un emploi public, 2612 individus, représentant 27367 jeunes appartiennent à la trajectoire "Fonctionnaire ».

Champ : échantillon "secteur public ».

Source : Génération 98.

\section{A - Trajectoires « stables»}

\section{A1 - Fonctionnaires}

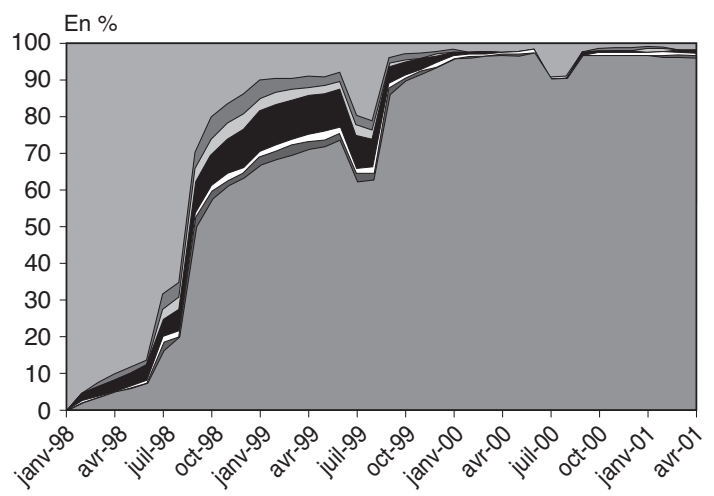

\section{A2 - CDI privé}

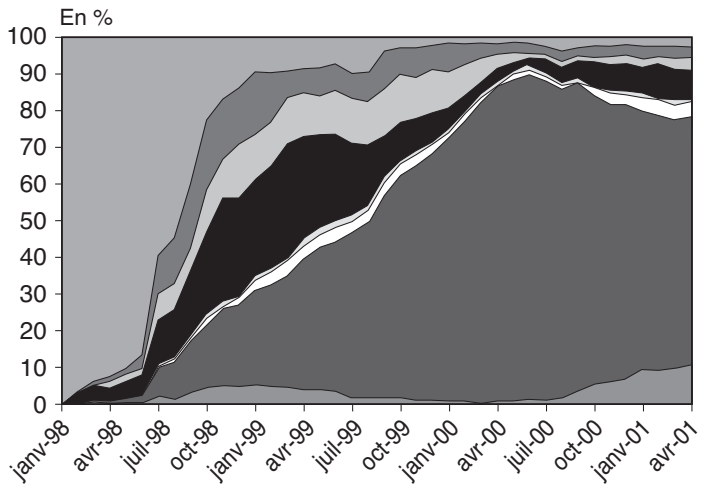

\section{B - Trajectoires « instables »}

\section{B1 - CDD public temporaires}

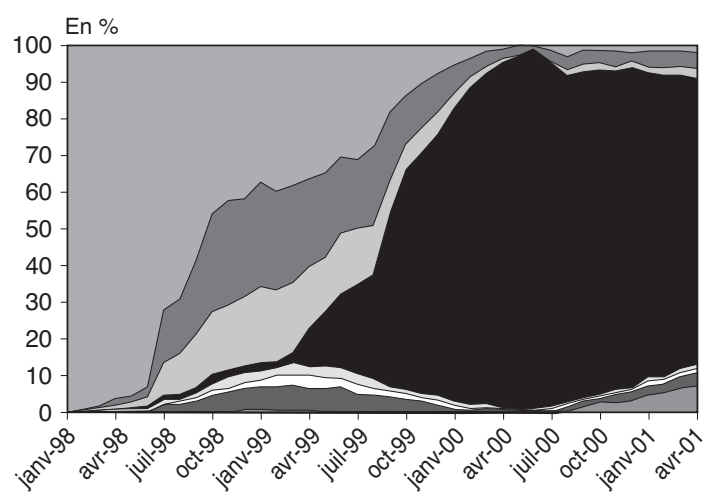

\section{B2 - CDD privé temporaires}

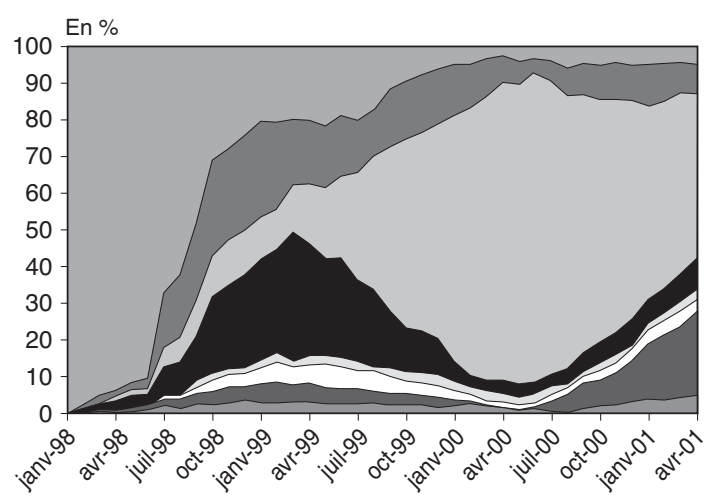

\begin{tabular}{|lll|}
\hline$\square$ Inactivé - Formation & $\square$ Chômage & $\square$ Contrat privé \\
Contrat public & $\square$ Mesures privé & $\square$ Mesures public \\
$\square$ CDI privé & $\square$ Fonctionnaire &
\end{tabular}




\section{C - Trajectoires " insertion dans la fonction publique "}

\section{C1 - Chômage}

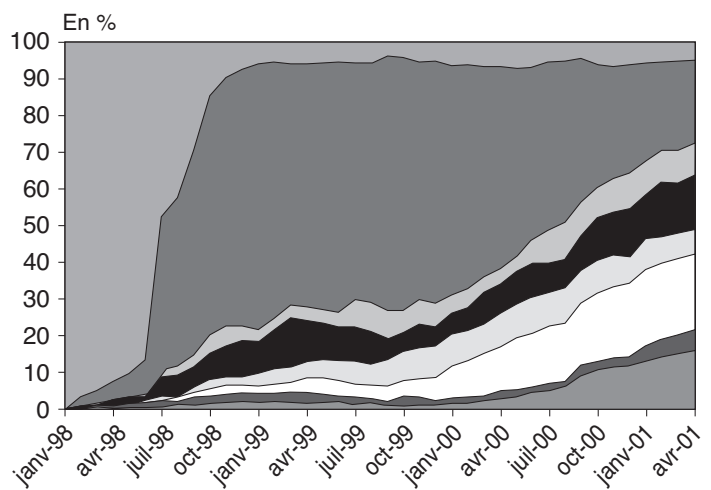

\section{C2 - Inactifs}

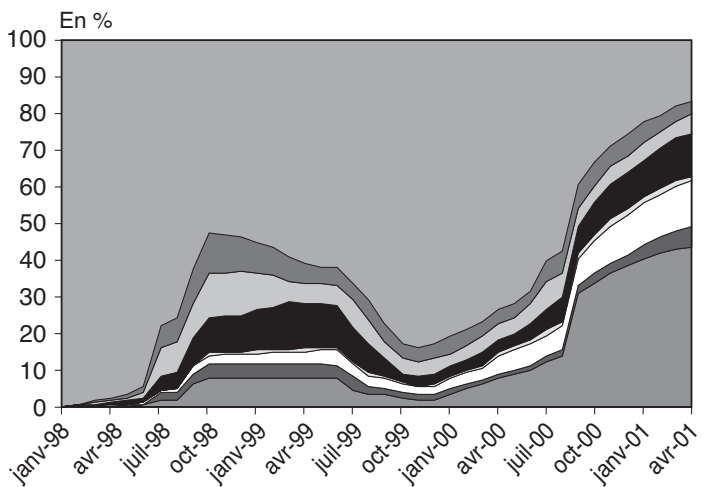

\section{C3 - CDD privé insérant}

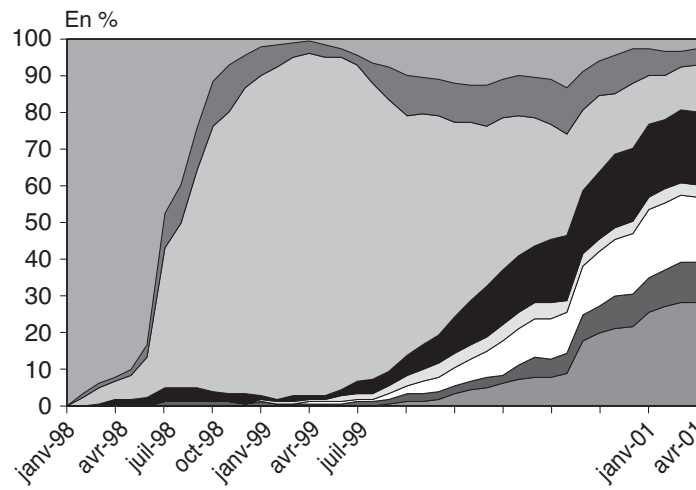

$\begin{array}{lll}\square \text { Inactivé - Formation } & \square \text { Chômage } & \square \text { Contrat privé } \\ \text { Contrat public } & \square \text { Mesures privé } & \square \text { Mesures public } \\ \square \text { CDI privé } & \square \text { Fonctionnaire } & \end{array}$

\section{D - Trajectoires « Stabilisation inconnue »}

\section{D1 - CDD public}

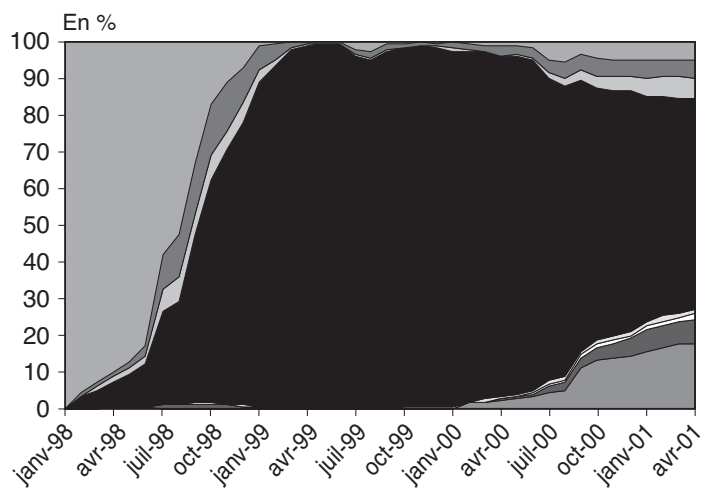

\section{D2 - Emploi jeunes}

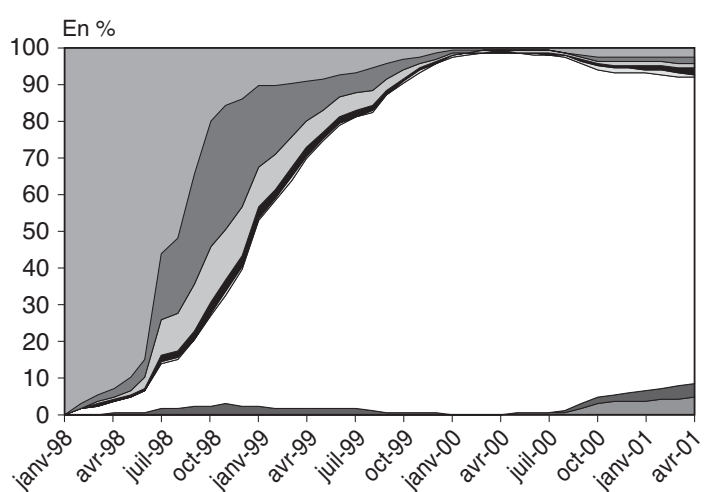

Champ : échantillon " secteur public ».

Source : Génération 98. 
Tableau A

Caractéristiques individuelles par trajectoires types

\begin{tabular}{|c|c|c|c|c|c|c|c|c|c|c|}
\hline \multirow{2}{*}{ Variables } & \multicolumn{9}{|c|}{ Trajectoires types (1) } & \multirow{2}{*}{$\begin{array}{c}\text { Échantillon } \\
\text { « secteur } \\
\text { public " }\end{array}$} \\
\hline & 1 & 2 & 3 & 4 & 5 & 6 & 7 & 8 & 9 & \\
\hline Homme & 39,25 & 43,23 & 42,31 & 54,02 & 65,82 & 57,42 & 35,69 & 37,62 & 31,94 & 40,62 \\
\hline \multicolumn{11}{|l|}{ Niveau de formation } \\
\hline Niveau I & 9,99 & 10,34 & 8,66 & 11,39 & 6,96 & 5,91 & 5,50 & 10,64 & 2,16 & 7,89 \\
\hline Niveau II & 53,75 & 15,29 & 12,69 & 24,37 & 17,77 & 32,39 & 18,21 & 30,31 & 18,87 & 29,78 \\
\hline Niveau III & 11,08 & 28,02 & 25,14 & 14,71 & 10,83 & 12,29 & 14,75 & 17,17 & 21,98 & 16,33 \\
\hline Niveau IV & 7,41 & 13,28 & 15,35 & 13,53 & 11,15 & 13,37 & 19,98 & 9,15 & 13,24 & 11,71 \\
\hline Niveau IV + & 8,57 & 12,96 & 14,09 & 15,88 & 16,42 & 22,08 & 18,90 & 13,97 & 32,75 & 17,07 \\
\hline Niveau V & 7,39 & 17,71 & 19,01 & 16,86 & 25,06 & 10,99 & 19,45 & 16,37 & 9,47 & 13,87 \\
\hline Niveau Vbis & 1,41 & 1,26 & 3,27 & 2,93 & 5,95 & 1,52 & 2,74 & 2,07 & 1,01 & 2,18 \\
\hline Niveau VI & 0,39 & 1,14 & 1,79 & 0,32 & 5,86 & 1,44 & 0,48 & 0,32 & 0,52 & 1,18 \\
\hline Vie en couple & 52,24 & 41,73 & 33,94 & 36,97 & 28,51 & 39,91 & 41,99 & 43,74 & 43,10 & 41,56 \\
\hline Avoir un enfant & 15,45 & 11,93 & 5,52 & 9,82 & 10,84 & 8,61 & 9,83 & 13,98 & 13,66 & 12,18 \\
\hline \multicolumn{11}{|l|}{ Situation du conjoint } \\
\hline Emploi public & 38,57 & 23,18 & 29,97 & 35,67 & 24,06 & 25,17 & 20,91 & 31,18 & 26,08 & 30,68 \\
\hline Emploi privé & 46,22 & 58,63 & 52,62 & 48,55 & 52,86 & 47,01 & 62,90 & 52,76 & 54,40 & 51,39 \\
\hline Inactif & 15,21 & 18,19 & 17,41 & 15,78 & 23,08 & 27,81 & 16,20 & 16,06 & 19,52 & 17,93 \\
\hline \multicolumn{11}{|l|}{$\begin{array}{l}\text { Situation du père } \\
\text { (fin étude) }\end{array}$} \\
\hline Emploi public & 27,50 & 25,84 & 26,26 & 26,67 & 27,45 & 30,27 & 28,56 & 25,33 & 25,84 & 27,05 \\
\hline Emploi privé & 47,47 & 53,83 & 53,92 & 50,22 & 42,47 & 52,21 & 50,33 & 49,25 & 48,36 & 49,11 \\
\hline Inactif & 25,03 & 20,33 & 19,82 & 23,10 & 30,08 & 17,51 & 21,10 & 25,42 & 25,80 & 23,84 \\
\hline \multicolumn{11}{|l|}{$\begin{array}{l}\text { Situation de la mère } \\
\text { (fin étude) }\end{array}$} \\
\hline Emploi public & 31,24 & 29,33 & 22,51 & 25,39 & 25,31 & 32,44 & 26,90 & 29,58 & 23,00 & 27,88 \\
\hline Emploi privé & 32,56 & 38,02 & 40,89 & 31,20 & 24,02 & 32,82 & 38,33 & 21,58 & 34,36 & 32,90 \\
\hline Inactive & 36,21 & 32,65 & 36,51 & 43,41 & 50,67 & 34,74 & 34,77 & 40,83 & 42,63 & 39,22 \\
\hline Âge médian en 1998 & 24 & 22 & 21 & 22 & 21 & 22 & 21 & 23 & 22 & 22 \\
\hline \multicolumn{11}{|l|}{ PCS en mars 2001} \\
\hline $\begin{array}{l}\text { Artisans, } \\
\text { commerçants, } \\
\text { chefs d'entreprise }\end{array}$ & 0,04 & 0,11 & 0,39 & 0,67 & 0,52 & 0,12 & - & 0,09 & 0,08 & 0,18 \\
\hline Cadres & 35,10 & 13,51 & 9,94 & 20,63 & 4,69 & 16,09 & 8,14 & 18,68 & 3,23 & 17,17 \\
\hline $\begin{array}{l}\text { Professions } \\
\text { intermédiaires }\end{array}$ & 37,01 & 30,79 & 20,55 & 31,32 & 22,39 & 26,15 & 29,68 & 38,49 & 61,90 & 36,23 \\
\hline Employés & 20,61 & 35,48 & 26,23 & 28,01 & 33,45 & 30,02 & 34,81 & 23,21 & 24,18 & 26,60 \\
\hline Ouvriers & 4,41 & 9,79 & 19,64 & 8,54 & 7,11 & 5,26 & 12,29 & 6,64 & 4,57 & 7,23 \\
\hline Effectifs (pondéré) & 27367 & 8425 & 7133 & 8337 & 10686 & 11420 & 7112 & 13883 & 17758 & 112121 \\
\hline
\end{tabular}

Lecture : parmi les individus passés au moins une fois par un emploi public et appartenant à la trajectoire « fonctionnaires », 39,2 \% sont des hommes.

Champ : échantillon " secteur public ».

Source : Génération 98. 
Tableau B

Parcours sur le marché du travail par trajectoire type

\begin{tabular}{|c|c|c|c|c|c|c|c|c|c|c|}
\hline \multirow{2}{*}{ Variables } & \multicolumn{9}{|c|}{ Trajectires types (1) } & \multirow{2}{*}{$\begin{array}{c}\text { Échantillon } \\
\text { « secteur } \\
\text { public» }\end{array}$} \\
\hline & 1 & 2 & 3 & 4 & 5 & 6 & 7 & 8 & 9 & \\
\hline \multicolumn{11}{|l|}{$\begin{array}{l}\text { Nombre de séquences } \\
\text { de chômage }\end{array}$} \\
\hline 0 & 85,33 & 47,22 & 28,35 & 44,09 & 1,12 & 64,02 & 51,67 & 69,91 & 48,89 & 55,76 \\
\hline 1 & 13,61 & 31,81 & 32,62 & 35,88 & 42,48 & 24,53 & 26,10 & 24,56 & 43,62 & 28,61 \\
\hline 2 & 0,82 & 12,51 & 20,03 & 15,40 & 31,02 & 0,21 & 12,53 & 3,82 & 6,39 & 9,73 \\
\hline 3 et plus & 0,24 & 8,46 & 19,00 & 4,63 & 25,38 & 2,24 & 9,70 & 1,70 & 1,11 & 5,90 \\
\hline \multicolumn{11}{|l|}{$\begin{array}{l}\text { Nombre de séquences } \\
\text { d'emploi }\end{array}$} \\
\hline 1 & 49,95 & - & - & 32,17 & 34,63 & 30,76 & - & 53,48 & 54,48 & 38,27 \\
\hline 2 & 31,33 & 40,17 & 26,43 & 41,38 & 32,89 & 44,91 & 35,64 & 33,55 & 32,91 & 34,76 \\
\hline 3 & 18,72 & 30,59 & 25,11 & 16,06 & 17,75 & 17,14 & 28,78 & 8,82 & 9,35 & 16,89 \\
\hline 4 et plus & - & 29,24 & 47,49 & 10,39 & 14,72 & 7,19 & 35,58 & 4,15 & 3,26 & 12,08 \\
\hline $\begin{array}{l}\text { Salaire médian } \\
\text { en mars } 2001\end{array}$ & 1400 & 1110 & 990 & 1040 & 885 & 1140 & 990 & 1060 & 885 & 1070 \\
\hline $\begin{array}{l}\text { Salaire médian à } \\
\text { l'embauche (2) }\end{array}$ & 220 & 1040 & 965 & 990 & 855 & 1130 & 965 & 975 & 855 & 990 \\
\hline $\begin{array}{l}\text { Ancienneté médiane dans } \\
\text { le dernier emploi (en mois) }\end{array}$ & 25 & 14 & 8 & 18 & 8 & 9 & 9 & 29 & 27 & 17 \\
\hline \multicolumn{11}{|l|}{ Situation en mars 2001} \\
\hline Fonctionnaire & 96,50 & 12,33 & 4,84 & 7,66 & 15,13 & 41,80 & 24,89 & 15,47 & 4,18 & 35,21 \\
\hline CDI privé & 0,91 & 65,71 & 20,85 & 2,63 & 5,93 & 5,66 & 9,73 & 6,13 & 3,41 & 9,74 \\
\hline CDD privé (3) & 0,53 & 1,72 & 31,68 & 2,16 & 8,48 & 4,79 & 14,61 & 6,03 & 1,62 & 6,49 \\
\hline CDD public (4) & 0,32 & 9,25 & 7,52 & 78,84 & 12,53 & 13,60 & 16,32 & 58,64 & 1,84 & 17,87 \\
\hline Mesures du secteur public & 0,26 & 4,62 & 3,53 & 1,61 & 21,88 & 13,54 & 20,64 & 1,38 & 83,73 & 19,27 \\
\hline Mesures du secteur privé & 0,27 & 0,94 & 3,38 & 0,74 & 7,03 & 1,19 & 3,99 & 2,41 & 0,74 & 2,09 \\
\hline Chômage & 0,35 & 3,74 & 8,02 & 3,95 & 22,75 & 3,66 & 4,06 & 4,11 & 1,78 & 4,76 \\
\hline Inactivité-Foermation & 1,09 & 1,66 & 4,17 & 2,38 & 5,47 & 15,61 & 2,97 & 5,62 & 2,65 & 4,33 \\
\hline \multicolumn{11}{|l|}{$\begin{array}{l}\text { Secteur d'emploi en } \\
\text { mars } 2001\end{array}$} \\
\hline Éducation nationale & 52,02 & 7,24 & 4,59 & 26,97 & 20,55 & 30,80 & 17,06 & 31,70 & 49,16 & \\
\hline Armées & 6,88 & 2,36 & 0,62 & 22,73 & 2,51 & 13,15 & 5,12 & 14,60 & 1,65 & 7,58 \\
\hline Autres État & 22,64 & 8,82 & 9,11 & 15,38 & 14,43 & 25,74 & 23,44 & 10,67 & 11,48 & 16,58 \\
\hline Collectivités territoriales & 16,66 & 9,86 & 5,29 & 28,58 & 29,30 & 14,93 & 21,93 & 26,05 & 30,95 & 21,08 \\
\hline Parapublic(5) & 0,04 & 1,73 & 2,43 & 0,40 & 3,43 & 1,19 & 2,48 & 2,40 & 0,31 & 1,37 \\
\hline Secter privé & 1,81 & 69,99 & 74,59 & 5,93 & 29,79 & 14,26 & 29,98 & 14,58 & 6,46 & 19,11 \\
\hline \multicolumn{11}{|l|}{ Durée cumulée } \\
\hline Fonctionnaire & 27,12 & 1,49 & 0,75 & 0,55 & 1,43 & 4,20 & 2,22 & 1,21 & 0,32 & 7,72 \\
\hline CDI du privé & 0,41 & 18,61 & 1,86 & 1,18 & 0,81 & 0,98 & 1,06 & 0,51 & 0,57 & 2,10 \\
\hline CDD du public & 1,23 & 3,46 & 3,86 & 16,75 & 2,50 & 2,58 & 2,85 & 26,43 & 0,45 & 6,07 \\
\hline CDD du privé & 0,50 & 2,44 & 10,45 & 1,89 & 1,60 & 1,37 & 11,52 & 0,65 & 0,85 & 2,35 \\
\hline Intérim du public & 0,01 & 0,42 & 0,25 & 0,46 & 0,17 & 0,03 & 0,11 & 0,43 & 0,01 & 0,16 \\
\hline Intérim du privé & 0,26 & 0,63 & 4,18 & 1,01 & 0,53 & 0,96 & 5,42 & 0,33 & 0,55 & 1,07 \\
\hline Emplois jeunes du public & 0,22 & 0,65 & 0,73 & 0,28 & 1,82 & 1,09 & 2,00 & 0,06 & 24,08 & 4,40 \\
\hline Emplois jeunes du privé & 0,08 & 0,19 & 0,14 & 0,18 & 1,44 & 0,10 & 0,48 & 0,25 & 0,08 & 0,27 \\
\hline CES & 0,08 & 0,10 & 0,37 & 0,06 & 1,57 & 0,30 & 0,13 & 0,02 & 1,11 & 0,40 \\
\hline $\begin{array}{l}\text { Apprentissage, } \\
\mathrm{CA}, \mathrm{CQ} \text { public }\end{array}$ & 0,13 & 0,09 & 0,14 & 0,04 & 0,21 & 0,36 & 0,29 & 0,05 & 0,49 & 0,21 \\
\hline $\begin{array}{l}\text { Aprentissage, } \\
\mathrm{CA}, \mathrm{CQ} \text { privé }\end{array}$ & 0,005 & 0,25 & 0,63 & 0,33 & 0,80 & 0,21 & 0,72 & 0,14 & 0,07 & $0 ., 27$ \\
\hline Chômage & 0,73 & 2,64 & 4,86 & 4,30 & 18,25 & 2,04 & 2,66 & 1,47 & 2,89 & 3,76 \\
\hline Inactivité & 1,59 & 1,23 & 1,68 & 2,24 & 0,96 & 6,23 & 1,32 & 0,97 & 0,95 & 1,83 \\
\hline Formation & 6,45 & 6,63 & 7,39 & 7,34 & 7,11 & 12,66 & 7,01 & 6,48 & 6,46 & 7,32 \\
\hline \multicolumn{11}{|c|}{$\begin{array}{l}\text { 1. Se reporter au tableau } 1 \text { pour la liste des trajectoires. } \\
\text { 2. Salaire d'embauche de la séquence d'emploi à la date d'enquête en euros (mars 2001). } \\
\text { 3. Y compris l'emploi intérimaire. } \\
\text { 4. Y compris l'emploi intérimaire. } \\
5 \text { Entreprises publiques ou nationalisées, école de la fonction publique et secteur hospitalier. }\end{array}$} \\
\hline
\end{tabular}

Lecture : parmi les individus passés au moins une fois par un emploi public et appartenant à la trajectoire « fonctionnaires ", 85,3 \% n'ont connu aucun épisode de chômage.

Champ : échantillon " secteur public ".

Source : Génération 98. 
ANNEXE 5

MODÈLE LOGIT MULTINOMIAL - APPARTENANCE AUX TRAJECTOIRES TYPES

(Log vraisemblance : - 16 834,4) $n=8$ 947. Trajectoire de référence : "fonctionnaires »

\begin{tabular}{|c|c|c|c|c|c|c|c|c|c|c|c|c|c|c|c|c|}
\hline \multirow{3}{*}{ Variables } & \multicolumn{16}{|c|}{ Trajectoires } \\
\hline & \multicolumn{2}{|c|}{ CDD privé instable } & \multicolumn{2}{|c|}{ Emplois jeunes } & \multicolumn{2}{|c|}{ Inactifs } & \multicolumn{2}{|c|}{ CDD public } & \multicolumn{2}{|c|}{ CDD privé insérant } & \multicolumn{2}{|c|}{ CDD public instable } & \multicolumn{2}{|c|}{ Chômage } & \multicolumn{2}{|c|}{ CDI privé } \\
\hline & Paramètres & P-value & Paramètres & P-value & Paramètres & P-value & Paramètres & P-value & Paramètres & P-value & Paramètres & P-value & Paramètres & P-value & Paramètres & P-value \\
\hline Constante & 0,5826 & 0,3811 & 1,0234 & 0,0539 & 4,7027 & 0,0000 & $-1,0243$ & 0,0220 & 2,3526 & 0,0018 & 1,9307 & 0,0021 & $-1,1575$ & 0,0435 & 1,6275 & 0,0097 \\
\hline Homme & $-0,2797$ & 0,0067 & $-0,5758$ & 0,0000 & 0,6308 & 0,0000 & $-0,2904$ & 0,0002 & $-0,2509$ & 0,0230 & 0,3122 & 0,0011 & $-0,744$ & 0,0000 & 0,0563 & 0,5705 \\
\hline Âge en 1998 & $-0,0788$ & 0,0011 & $-0,1214$ & 0,0000 & $-0,2532$ & 0,0000 & 0,0227 & 0,1565 & $-0,1563$ & 0,0000 & $-0,1317$ & 0,0000 & $-0,0221$ & 0,2800 & $-0,1003$ & 0,0000 \\
\hline \multicolumn{17}{|l|}{$\begin{array}{l}\text { Niveau de } \\
\text { formation }\end{array}$} \\
\hline Niveau I & Réf. & Réf. & Réf. & Réf. & Réf. & Réf. & Réf. & Réf. & Réf. & Réf. & Réf. & Réf. & Réf. & Réf. & Réf. & Réf. \\
\hline Niveau II & $-1,3176$ & 0,0000 & 0,2862 & 0,0955 & $-0,0553$ & 0,7218 & $-0,7957$ & 0,0000 & $-0,9568$ & 0,0000 & $-1,0954$ & 0,0000 & $-0,7955$ & 0,0000 & $-1,4426$ & 0,0000 \\
\hline Niveau III & 0,8387 & 0,0000 & 1,9438 & 0,0000 & 0,1245 & 0,5300 & 0,404 & 0,0047 & 0,4917 & 0,0254 & 0,1186 & 0,5161 & 0,4171 & 0,0244 & 0,6654 & 0,0001 \\
\hline Niveau IV + & 0,6513 & 0,0032 & 2,6794 & 0,0000 & 0,8464 & 0,0000 & 0,7361 & 0,0000 & 0,7896 & 0,0010 & 0,308 & 0,1313 & 1,1063 & 0,0000 & 0,3231 & 0,1252 \\
\hline Niveau IV & 0,5257 & 0,0356 & 1,5999 & 0,0000 & $-0,0439$ & 0,8540 & 0,3816 & 0,0549 & 0,6513 & 0,0152 & $-0,0133$ & 0,9552 & 0,7987 & 0,0006 & 0,1028 & 0,6707 \\
\hline Niveau V & 0,6304 & 0,0158 & 1,2361 & 0,0000 & $-0,6565$ & 0,0105 & 0,9481 & 0,0000 & 0,5511 & 0,0549 & 0,06 & 0,8056 & 1,637 & 0,0000 & 0,2535 & 0,3058 \\
\hline Niveau V bis & 0,9304 & 0,0144 & 1,1045 & 0,0035 & $-0,9149$ & 0,0236 & 0,8637 & 0,0092 & 0,6282 & 0,1265 & 0,0223 & 0,9528 & 2,1912 & 0,0000 & $-0,2857$ & 0,5146 \\
\hline \multicolumn{17}{|l|}{$\begin{array}{l}\text { Lieu de résidence } \\
\text { fin études }\end{array}$} \\
\hline Paris-Ile-de-France & Réf. & Réf. & Réf. & Réf. & Réf. & Réf. & Réf. & Réf. & Réf. & Réf. & Réf. & Réf. & Réf. & Réf. & Réf. & Réf. \\
\hline Province & 0,5284 & 0,0005 & 0,4611 & 0,0001 & 0,1283 & 0,2775 & 0,0331 & 0,7358 & 0,1055 & 0,4568 & 0,2686 & 0,0408 & 0,7489 & 0,0000 & $-0,28751$ & 0,0155 \\
\hline Vie en couple & $-0,3491$ & 0,0064 & $-0,1249$ & 0,1889 & $-0,4885$ & 0,0000 & $-0,1523$ & 0,1075 & $-0,0198$ & 0,8790 & $-0,1549$ & 0,2008 & $-0,6625$ & 0,0000 & 0,2198 & 0,0612 \\
\hline \multicolumn{17}{|l|}{\begin{tabular}{|l} 
Situation du \\
conjoint mars 2001
\end{tabular}} \\
\hline Secteur privé & Réf. & Réf. & Réf. & Réf. & Réf. & Réf. & Réf. & Réf. & Réf. & Réf. & Réf. & Réf. & Réf. & Réf. & Réf. & Réf. \\
\hline Secteur public & $-0,4898$ & 0,0066 & $-0,4009$ & 0,0015 & $-0,4228$ & 0,0075 & $-0,4228$ & 0,0318 & $-0,7114$ & 0,0002 & $-0,3717$ & 0,0182 & $-0,3391$ & 0,0410 & $-0,6470$ & 0,0000 \\
\hline Inactif & 0,1348 & 0,5133 & 0,1518 & 0,3135 & 0,3458 & 0,0443 & 0,3458 & 0,1950 & $-0,1146$ & 0,5982 & $-0,1085$ & 0,5794 & 0,3783 & 0,0432 & $-0,0309$ & 0,8648 \\
\hline \multicolumn{17}{|l|}{$\begin{array}{l}\text { Situation du père } \\
\text { en } 1998\end{array}$} \\
\hline Secteur privé & Réf. & Réf. & Réf. & Réf. & Réf. & Réf. & Réf. & Réf. & Réf. & Réf. & Réf. & Réf. & Réf. & Réf. & Réf. & Réf. \\
\hline Secteur public & $-0,0913$ & 0,4402 & $-0,1549$ & 0,0915 & $-0,0578$ & 0,5508 & $-0,2970$ & 0,0013 & $-0,1659$ & 0,1863 & $-0,2423$ & 0,0326 & $-0,1501$ & 0,1691 & $-0,2727$ & 0,0169 \\
\hline Inactif & 0,0003 & 0,9982 & 0,1468 & 0,1216 & $-0,1446$ & 0,1912 & $-0,1364$ & 0,8823 & 0,0474 & 0,7225 & 0,0701 & 0,5430 & 0,3215 & 0,0027 & $-0,2320$ & 0,0640 \\
\hline Secteur public & $-0,4845$ & 0,0065 & $-0,3595$ & 0,0003 & $-0,0091$ & 0,9306 & 0,0205 & 0,8335 & $-0,2781$ & 0,0386 & $-0,1031$ & 0,3961 & 0,0817 & 0,4961 & $-0,0205$ & 0,8643 \\
\hline Inactive & $-0,3115$ & 0,0002 & 0,0020 & 0,9812 & $-0,1081$ & 0,2871 & 0,0595 & 0,5104 & $-0,1916$ & 0,1172 & 0,0353 & 0,7479 & 0,2571 & 0,0167 & $-0,1217$ & 0,2887 \\
\hline
\end{tabular}

Champ : échantillon « secteur public ».

Source : Génération 98. 
ANNEXE 6

PROBABILITÉ D'OCCUPER UN CDI OU D'ÊTRE FONCTIONNAIRE EN MARS 2001 ÉTANT DONNÉ LA PROBABILITÉ DE PASSAGES ANTÉRIEURS EN EMPLOI NON PÉRENNE DU SECTEUR PUBLIC OU PRIVÉ (MODĖLES PROBIT TRIVARIÉ)

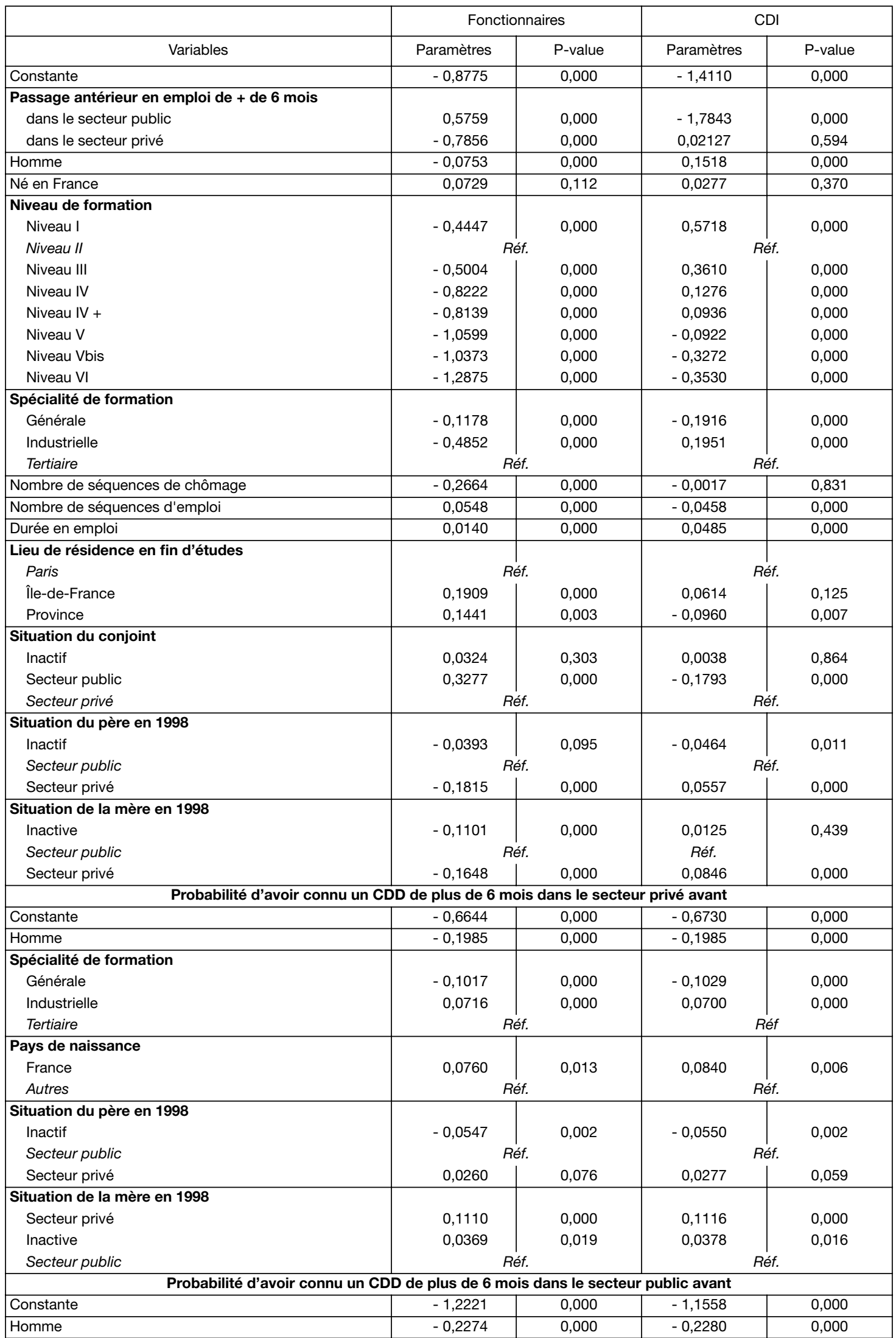




\begin{tabular}{|c|c|c|c|c|}
\hline \multirow[b]{2}{*}{ Variables } & \multicolumn{2}{|c|}{ Fonctionnaires } & \multicolumn{2}{|c|}{ CDI } \\
\hline & Paramètres & P-value & Paramètres & P-value \\
\hline $\begin{array}{l}\text { Spécialité de formation } \\
\text { Générale } \\
\text { Industrielle } \\
\text { Tertiaire }\end{array}$ & $\begin{array}{r}0,0673 \\
-0,4804\end{array}$ & $\begin{array}{l}0,000 \\
0,000\end{array}$ & $\begin{array}{c}0,0713 \\
-0,4673\end{array}$ & $\begin{array}{l}0,000 \\
0,000\end{array}$ \\
\hline $\begin{array}{l}\text { Pays de naissance } \\
\text { France } \\
\text { Autres }\end{array}$ & 0,2247 & 0,000 & 0,1663 & 0,001 \\
\hline $\begin{array}{l}\text { Pays de naissance du père } \\
\text { France }\end{array}$ & \multicolumn{2}{|c|}{ Réf. } & \multicolumn{2}{|c|}{ Réf. } \\
\hline $\begin{array}{l}\text { Situation du père en } 1998 \\
\text { Inactif } \\
\text { Secteur public } \\
\text { Secteur privé }\end{array}$ & $\begin{array}{r}0,0886 \\
-0,0127\end{array}$ & $\begin{array}{r}0,000 \\
\text { Réf. } \\
0,507\end{array}$ & $\begin{array}{r}0,0850 \\
-0,0234\end{array}$ & 0,223 \\
\hline $\begin{array}{l}\text { Situation de la mère en } 1998 \\
\text { Secteur privé } \\
\text { Inactive } \\
\text { Secteur public }\end{array}$ & $\begin{array}{l}-0,1514 \\
-0,1335\end{array}$ & $\begin{array}{l}0,000 \\
0,000\end{array}$ & $\begin{array}{l}-0,1537 \\
-0,1371\end{array}$ & $\begin{array}{l}0,000 \\
0,000\end{array}$ \\
\hline $\begin{array}{l}\text { Coefficients de corrélation } \\
\rho_{12}=\operatorname{corr}\left(\varepsilon_{1 i}, \varepsilon_{2 i}\right) \\
\rho_{13}=\operatorname{corr}\left(\varepsilon_{1 i}, \varepsilon_{3 i}\right) \\
\rho_{23}=\operatorname{corr}\left(\varepsilon_{2 i}, \varepsilon_{3 i}\right)\end{array}$ & $\begin{array}{r}0,0190 \\
-0,0007 \\
-0,2091\end{array}$ & $\begin{array}{l}0,172 \\
0,983 \\
0,000\end{array}$ & $\begin{array}{c}-0,0488 \\
0,1551 \\
-0,2444\end{array}$ & $\begin{array}{l}0,048 \\
0,008 \\
0,000\end{array}$ \\
\hline Log vraisemblance & \multicolumn{2}{|c|}{$-60085,451$} & \multicolumn{2}{|c|}{$-76789,905$} \\
\hline $\mathbf{N}$ & \multicolumn{4}{|c|}{$53781(1)$} \\
\hline Test LR $\rho_{12}=\rho_{13}=\rho_{23}=0$ & $\begin{array}{r}434,40 \\
9\end{array}$ & 0,000 & 504,154 & 0,000 \\
\hline
\end{tabular}

Champ : Échantillon total.

Source : Génération 98. 